\title{
No-Pain No-Gain: DRL Assisted Optimization in Energy-Constrained CR-NOMA Networks
}

\author{
Zhiguo Ding, Fellow, IEEE, Robert Schober, Fellow, IEEE, and H. Vincent Poor, \\ Life Fellow, IEEE
}

\begin{abstract}
This paper applies machine learning to optimize the transmission policy of cognitive radio inspired non-orthogonal multiple access (CR-NOMA) networks, where time-division multiple access (TDMA) is used to serve multiple primary users and an energy-constrained secondary user is admitted to the primary users' time slots via NOMA. During each time slot, the secondary user performs the two tasks: data transmission and energy harvesting based on the signals received from the primary users. The goal of the paper is to maximize the secondary user's long-term throughput, by optimizing its transmit power and the time-sharing coefficient for its two tasks. The long-term throughput maximization problem is challenging due to the need for making decisions that yield long-term gains but might result in shortterm losses. For example, when in a given time slot, a primary user with large channel gains transmits, intuition suggests that the secondary user should not carry out data transmission due to the strong interference from the primary user but perform energy harvesting only, which results in zero data rate for this time slot but yields potential long-term benefits. In this paper, a deep reinforcement learning (DRL) approach is applied to emulate this intuition, where the deep deterministic policy gradient (DDPG) algorithm is employed together with convex optimization. Our simulation results demonstrate that the proposed DRL assisted NOMA transmission scheme can yield significant performance gains over two benchmark schemes.
\end{abstract}

\section{INTRODUCTION}

Machine learning has been recognized as one of the most important enabling technologies for the next generation wireless networks [1]. The key idea behind machine learning is to learn to make optimal decisions based on observed data or the environment [2]. Because of its general

Z. Ding and H. V. Poor are with the Department of Electrical Engineering, Princeton University, Princeton, NJ 08544, USA. Z. Ding is also with the School of Electrical and Electronic Engineering, the University of Manchester, Manchester, UK (email: zhiguo.ding@manchester.ac.uk. poor@princeton.edu). R. Schober is with the Institute for Digital Communications, FriedrichAlexander-University Erlangen-Nurnberg (FAU), Germany (email: robert.schober@ fau.de). 
utility, machine learning has been applied to a variety of wireless communication problems. For example, supervised learning has been applied to link adaption and channel estimation in orthogonal frequency-division multiplexing (OFDM) systems [3], [4]. Unsupervised learning has been shown to be particularly beneficial for improving the accuracy of wireless positioning in [5] and reducing the complexity of beamformer design [6]. Reinforcement learning has also been shown to be applicable to various communication problems, including energy harvesting, resource allocation, data and computation offloading, and network security [7]-[9].

This paper considers the application of machine learning to non-orthogonal multiple access (NOMA) systems. We note that this application has already received significant attention in the literature [10]. For example, an unsupervised machine learning algorithm, K-means, has been applied to millimeter-wave NOMA networks for the joint design of beamforming and user clustering [11]. A similar K-means based approach has also been proposed for terahertz NOMA systems employing nodes equipped with multiple antennas [12]. A power minimization problem for multi-carrier NOMA facilitated by simultaneous wireless information and power transfer (SWIPT) has been solved by applying deep learning in [13]. In [14], an intelligent offloading scheme powered by deep learning was proposed for NOMA assisted mobile edge computing (NOMA-MEC). Furthermore, deep learning approaches have been developed for the design of NOMA transceivers and the codebook of sparse code multiple access (SCMA) in [15] and [16], respectively. An overview of additional related work on the application of machine learning to NOMA can be found in recent survey articles [17]-[20].

This paper focuses on a cognitive radio (CR) inspired NOMA network, where multiple primary users and one energy-constrained secondary user communicate with the same base station, as shown in Fig. 1(a), Time-division multiple access (TDMA) is used to serve the primary users, where the use of CR-NOMA ensures that the secondary user can be admitted to the primary users' time slots without degrading their quality of service (QoS) experience [21]. During each time slot, the secondary user performs the two tasks: Data transmission and energy harvesting from the signals sent by the primary users [22]. We note that even if all the users' channels are constant, for the secondary user, its communication environment is time-varying and changes from one time slot to the next, since different primary users are scheduled in different time slots, as shown in Fig. 1(b), This time-varying pattern is difficult to handle with conventional optimization tools, but can be effectively learned by machine learning.

The objective of this paper is to maximize the secondary user's long-term throughput, by 
optimizing its transmit power and also the time-sharing coefficient for its two tasks. This throughput maximization problem is challenging because of the need for making decisions which might yield a long-term gain but could result in a short-term loss. For example, when a primary user with strong channels to both the base station and the secondary user transmits in a given time slot, a human would decide that the secondary user should not transmit data but carry out energy harvesting only, since the primary user would cause severe interference. Even though this decision leads to zero data rate in this particular time slot, it may yield a performance gain in the future since a significant amount of energy can be harvested due to the primary user's strong channel conditions. With conventional optimization tools, it is difficult to emulate this "no-pain no-gain'-like decision, which motivates the application of machine learning to the considered NOMA scenario, because machine learning algorithms are well known for their capability of emulating human behaviour and making 'no-pain no-gain'-like decisions [2], [23], [24].

Recall that compared to supervised and unsupervised machine learning, reinforcement learning has the capability to make decisions and perform learning at the same time, which is a desirable feature for wireless communication applications [7], [23], [25], [26]. Deep deterministic policy gradient (DDPG) is an effective implementation of deep reinforcement learning (DRL), where the action-value functions are approximated by neural networks and the action space does not have to be discrete [27], [28]. Because the optimization variables for the considered long-term throughput maximization problem are continuous, DDPG is adopted in this paper. In order to facilitate the application of DDPG, the considered long-term throughput maximization problem is first decomposed into two optimization subproblems. The first subproblem focuses on a single time slot and optimizes the secondary user's transmit power and the time sharing coefficient, where the closed-form optimal solution is found by applying convex optimization. The second subproblem targets the optimization of the energy fluctuation across different time slots, and is solved by applying the adopted DRL tool, DDPG. Extensive simulation results are provided to demonstrate that the proposed DDPG assisted NOMA scheme effectively emulates human decisions and offers significant performance gains over two benchmark schemes.

\section{SySTEM MODEL}

Consider a CR-NOMA uplink scenario as shown in Fig. 1(a), with one base station, $M$ primary users, denoted by $\mathrm{U}_{m}, 1 \leq m \leq M$, and one energy-constrained secondary user, denoted by $\mathrm{U}_{0}$. It is assumed that the $M$ primary users are scheduled to transmit in different time slots as 


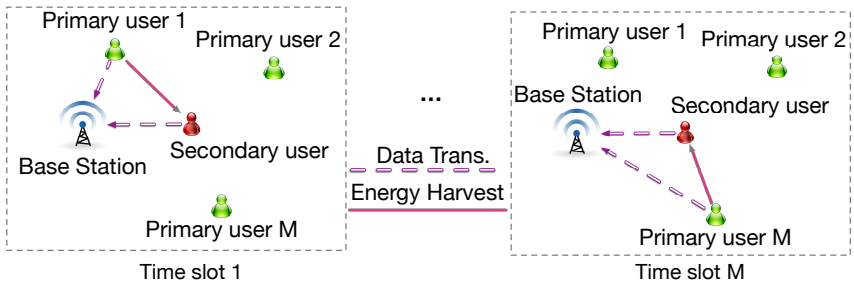

(a) System Model

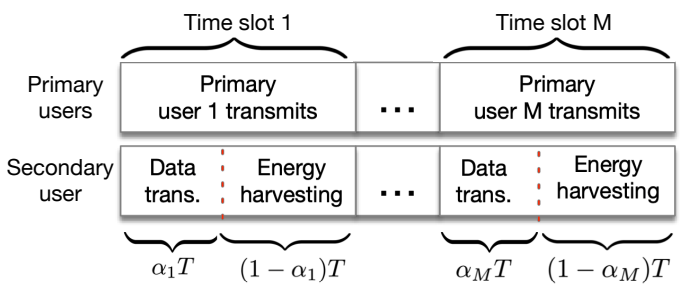

(b) CR-NOMA Transmission

Fig. 1. System diagram for the considered CR-NOMA uplink communication scenario, where a secondary user shares the spectrum with $M$ primary users and harvests energy from the signals sent by the primary users.

shown in Fig. 1(b), and this TDMA based scheduling is fixed and carried out over a long period of time such that each primary user is scheduled to transmit multiple times 1 . In particular, in the $n$-th time slot, denoted by $t_{n}$, user $\mathrm{U}_{m}, m=(n-1) \oplus M+1$, is scheduled to transmit, where $\oplus$ denotes the modulo operation. For example, for $M=2$, the primary users scheduled at $t_{1}, t_{2}, t_{3}$, and $t_{4}$ are $\mathrm{U}_{1}, \mathrm{U}_{2}, \mathrm{U}_{1}$, and $\mathrm{U}_{2}$, respectively. For notational convenience, denote the primary user scheduled at $t_{n}$ by $\overline{\mathrm{U}}_{n}$, i.e., $\overline{\mathrm{U}}_{n}=\mathrm{U}_{m}, m=(n-1) \oplus M+1$. Denote the channel gain between the base station and $\overline{\mathrm{U}}_{n}$ by $h_{n}$, the channel between $\mathrm{U}_{0}$ and $\overline{\mathrm{U}}_{n}$ by $h_{n, 0}$, and the channel between $\mathrm{U}_{0}$ and the base station at $t_{n}$ by $g_{0, n}$, where the effects of both large-scale path loss and quasi-static multi-path fading are included.

The secondary user is admitted to the primary users' time slots via CR-NOMA. In particular, in time slot $t_{n}$, the secondary user uses the first $\alpha_{n} T$ seconds for its data transmission, where $\alpha_{n}$ is a time-sharing parameter, $0 \leq \alpha_{n} \leq 1$, and $T$ denotes the duration of each time slot. The remainder of the time slot, $\left(1-\alpha_{n}\right) T$ seconds, will be used for battery charging by harvesting energy from the signals sent by primary user $\overline{\mathrm{U}}_{n}$, as shown in Fig. 1(b), At the beginning of $t_{n}$, the scheduled primary user's channel state information (CSI), i.e., $h_{n}$ and $h_{n, 0}$, is assumed to be available at the secondary user2.

Denote the remaining energy in the secondary user's battery at the beginning of the $n$-th time

${ }^{1}$ We note that the key idea of the proposed NOMA scheme is to ensure that the admission of the secondary user as well as the proposed resource allocation for the secondary user are transparent to the primary users. In other words, we do not adjust the primary users' transmission strategies because it is not the primary users' responsibility to accommodate the secondary user.

${ }^{2}$ The required CSI knowledge can be realized as follows. Prior to its transmission, primary user $\overline{\mathrm{U}}_{n}$ can broadcast a pilot signal which allows the base station to estimate $h_{n}$ and the secondary user to estimate $h_{n, 0}$ simultaneously. Via a reliable feedback link, the base station can pass its knowledge of $h_{n}$ to the secondary user. The base station needs to further broadcast a pilot signal and to enable the secondary user to estimate $g_{0, n}$. For low-mobility applications, such as Internet of Things with static sensors, the system overhead caused by channel estimation is moderate since the pilot signals can be sent infrequently. 
slot by $E_{n}$, which means that $E_{n+1}$ can be expressed as follows:

$$
E_{n+1}=\min \{\underbrace{\left(1-\alpha_{n}\right) T \eta P_{n}\left|h_{n, 0}\right|^{2}}_{\text {Harvested energy }}-\underbrace{\alpha_{n} T P_{0, n}}_{\text {Used energy }}+E_{n}, E_{\max }\},
$$

where $E_{\max }$ denotes the capacity limit of the secondary user's battery, $\eta$ denotes the energy harvesting efficiency coefficient, $P_{n}$ and $P_{0, n}$ denote the transmit powers of $\overline{\mathrm{U}}_{n}$ and $\mathrm{U}_{0}$ at $t_{n}$, respectively. Assume that the initial amount of energy available in the secondary user's battery is $E_{\max }$, i.e., $E_{1}=E_{\max }$. We note that (1) ensures that the amount of harvested energy cannot exceed the battery capacity, $E_{\max }$.

Due to the energy causality constraint, the secondary user's transmit power is capped by the energy available in its battery, i.e.,

$$
\alpha_{n} T P_{0, n} \leq E_{n}
$$

We note that (2) ensures that $E_{n+1}$ shown in (1) is always non-negative.

Therefore, the data rate that the secondary user can achieve at $t_{n}$ is given by 3

$$
R_{n}=\alpha_{n} \ln \left(1+\frac{P_{0, n}\left|g_{0, n}\right|^{2}}{1+P_{n}\left|h_{n}\right|^{2}}\right), \text { [nats per channel use (NPCU)], }
$$

which guarantees that the secondary user's signal can be successfully decoded during the first stage of successive interference cancellation (SIC) at the base station. After the base station removes the successfully decoded signal of the secondary user from the received signal, the primary users' signals can be decoded in the same manner as when the secondary user is absent. In other words, (3) guarantees that the primary users' QoS experience is not affected by admitting the secondary user into their time slots. We note that (3) is based on the QoS-based SIC decoding order, but more sophisticated SIC strategies can be used to further improve the performance of the CR-NOMA scheme, as discussed in [29].

The aim of this paper is to maximize the secondary user's long-term throughput by optimizing the time-sharing parameter, $\alpha_{n}$, and the secondary user's transmit power, $P_{0, n}$, in the $n$-th time slot $t_{n}$. Define the secondary user's transmission environment at $t_{n}$, also termed the state in the context

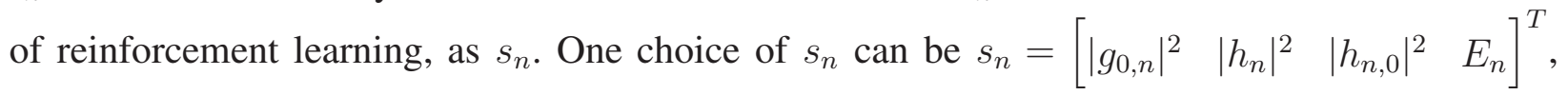
where $[\cdot]^{T}$ denotes a transpose operation. Define the policy which is a strategy, i.e., a set of

\footnotetext{
${ }^{3}$ For notational convenience, the noise power is assumed to be normalized, such that the value of the noise power is absorbed into the channel gains $\left|g_{0, n}\right|^{2}$ and $\left|h_{n}\right|^{2}$.
} 
sequential actions $\left[P_{0,1}, \alpha_{1}, P_{0,2}, \alpha_{2}, \ldots\right]$, adopted by the secondary user as $\pi_{P_{0, n}, \alpha_{n}}$. Then, the corresponding optimization problem can be formulated as follows [8], [9], [23]:

$$
\begin{array}{rl}
\max _{\pi_{P_{0}, n}, \alpha_{n}} & \mathcal{E}\left\{\sum_{n=1}^{\infty} \gamma^{n-1} R_{n} \mid \pi_{P_{0, n}, \alpha_{n}}, s_{1}\right\} \\
\text { s.t. } & E_{n+1}=\min \left\{\left(1-\alpha_{n}\right) T \eta P_{n}\left|h_{n, 0}\right|^{2}-\alpha_{n} T P_{0, n}+E_{n}, E_{\max }\right\} \\
& \alpha_{n} T P_{0, n} \leq E_{n} \\
& 0 \leq \alpha_{n} \leq 1 \\
& 0 \leq P_{0, n} \leq P_{\max },
\end{array}
$$

where $\mathcal{E}\left\{\sum_{n=1}^{\infty} \gamma^{n-1} R_{n} \mid \pi_{P_{0, n}, \alpha_{n}}, s_{1}\right\}$ denotes the expected value of the discounted cumulative sum of the data rates for given $\pi_{P_{0, n}, \alpha_{n}}$ and $s_{1}$, also termed the state-value function for policy $\pi_{P_{0, n}, \alpha_{n}}$ in the context of reinforcement learning 4 , $P_{\max }$ denotes the maximal transmit power of the secondary user, and $\gamma$ denotes the discount rate parameter [23]. We note that the use of the discount rate parameter ensures that a decision which yields a long-term gain is preferred over a short-sighted decision which maximizes the instantaneous data rate only (i.e., $\gamma=0$ ). In other words, by varying the choice of $\gamma$ between 0 and 1, a different tradeoff between the long-term gain and instantaneous benefit can be achieved. We note that $\gamma$ has to be strictly smaller than one, which is to ensure that the infinite sum in the objective function of $\mathrm{P} 1$ is finite.

Remark 1: One can also formulate the problem by asking the secondary user to charge its battery in each time slot before transmitting its data. Intuitively, both formulations should lead to the same problem formulation, which however is not true. In particular, if the secondary user carries out energy harvesting first, the energy available for data transmission is given by

$$
\min \left\{E_{\max }, E_{n}+\alpha_{n} T \eta P_{n}\left|h_{n, 0}\right|^{2}\right\}
$$

which means that the constraints in $(\underline{\mathrm{P} 1 \mathrm{c})}$ ) and $(\underline{\mathrm{P} 1 \mathrm{~d}})$ need to be formulated as follows:

$$
\begin{aligned}
E_{n+1} & =\max \left\{\min \left\{E_{\max }, E_{n}+\alpha_{n} T \eta P_{n}\left|h_{n, 0}\right|^{2}\right\}-\left(1-\alpha_{n}\right) T P_{0, n}, 0\right\}, \\
\alpha_{n} T P_{0, n} & \leq \min \left\{E_{\max }, E_{n}+\alpha_{n} T \eta P_{n}\left|h_{n, 0}\right|^{2}\right\},
\end{aligned}
$$

\footnotetext{
${ }^{4}$ The expectation carried out in $\mathrm{P1a}$ is explained in the following. In particular, given the policy, the secondary user's action at $t_{n}, n \geq 1$, is fixed. However, even though both $s_{1}$ and the actions are fixed, the realization of the state and the secondary user's data rate at $t_{n}, n>1$, are not deterministic due to the time-varying environment. Hence, the expectation is needed to capture the statistical property of the state transitions.
} 
respectively. The constraints in (5) and (6) are more complicated than $(\underline{\mathrm{P} 1 \mathrm{c}})$ and $(\underline{\mathrm{P} 1 \mathrm{~d}})$, which makes solving the rate maximization problem more difficult.

Remark 2: The following two strategies will be used as benchmark schemes in this paper. For the first scheme, termed the greedy algorithm, $U_{0}$ uses all the energy available for data transmission, and then starts energy harvesting. In particular, $\mathrm{U}_{0}$ 's transmit power is fixed at $P_{\max }$, and $\alpha_{n}=\min \left\{1, \frac{E_{n}}{T P_{\max }}\right\}$ is adopted by the greedy algorithm. For the case that $T P_{\max } \geq E_{n}$, this choice of $\alpha_{n}$ means that all the energy available, $E_{n}$, will be used to power data transmission, i.e., $T P_{\max } \alpha_{n}=E_{n}$. For the second benchmark scheme, termed the random algorithm, $P_{\max }$ is used as $\mathrm{U}_{0}$ 's transmit power and $\alpha_{n}$ is uniformly generated between 0 and $\min \left\{1, \frac{E_{n}}{T P_{\max }}\right\}$. Because $\alpha_{n} \leq \frac{E_{n}}{T P_{\max }}$, it is guaranteed that there is enough energy for $\mathrm{U}_{0}$ to transmit during $\alpha_{n} T$ seconds with transmit power $P_{\max }$.

Remark 3: Problem P1 cannot be straightforwardly solved by applying conventional convex optimization, not only because its equality constraint in ( $\mathrm{P1b}$ is not affine, but also because its objective function is a long-term non-convex throughput function. However, Problem P1 is ideally suited for the application of machine learning, as discussed in the following section.

\section{Problem Reformulation for Application of Reinforcement Learning}

\section{A. Rationale Behind the Application of Reinforcement Learning}

For the considered optimization problem, 'no-pain no-gain' decisions, i.e., decisions which result in short-term losses but yield long-term gains, have to be made. These decisions motivate the application of reinforcement learning. In the following, a simple example with two primary users, i.e., $\mathrm{U}_{1}$ and $\mathrm{U}_{2}$, is used to illustrate the 'no-pain no-gain' situations inherent to the considered CR-NOMA scenario.

In particular, assume that $\mathrm{U}_{1}$ has very strong channels to both $\mathrm{U}_{0}$ and the base station, i.e., $\left|h_{1}\right|^{2} \rightarrow \infty$ and $\left|h_{1,0}\right|^{2} \rightarrow \infty$, whereas $U_{2}$ has very weak channels to both $U_{0}$ and the base station, i.e., $\left|h_{2}\right|^{2} \rightarrow 0$ and $\left|h_{2,0}\right|^{2} \rightarrow 0$. Intuition in this situation suggests to encourage the secondary user to do the following:

- Use most of the time slot for energy harvesting when $U_{1}$ transmits. The reason is that $\left|h_{1}\right|^{2} \rightarrow \infty$ means that $\mathrm{U}_{1}$ is a strong interference source for $\mathrm{U}_{0}$ 's data transmission, and $\left|h_{1,0}\right|^{2} \rightarrow \infty$ means an opportunity to harvest a large amount of energy from $U_{1}$.

- Use most of the time slot for data transmission when $\mathrm{U}_{2}$ transmits. The reason is that $\left|h_{2}\right|^{2} \rightarrow 0$ implies an interference free situation, and hence it is possible to achieve a high 
data rate, as indicated by (3). $\left|h_{1,0}\right|^{2} \rightarrow 0$ implies that only a small amount of energy can be harvested from $\mathrm{U}_{2}$.

Note that the actions following this intuition might incur short-term losses compared to the benchmark schemes. For example, when $\mathrm{U}_{1}$ transmits, a rationale action is to ask $\mathrm{U}_{0}$ to carry out energy harvesting only, which leads to zero data rate in this particular time slot. In other words, in the time slots where $\mathrm{U}_{1}$ transmits, the data rate achieved by the action following the intuition is smaller than the data rates achieved by the benchmark schemes. However, this temporary sacrifice yields a large amount of harvested energy and hence a potential long-term gain. Reinforcement learning is an ideal tool for emulating this intuition by learning the feature inherent to the considered problem, as shown in the remainder of this paper.

\section{B. Problem Reformulation}

For the throughput maximization problem formulated in $\mathrm{P1}$, there are two sets of optimization variables, namely $P_{0, n}$ and $\alpha_{n}$, and the value ranges of $P_{0, n}$ and $\alpha_{n}$ can be quite different, i.e., $0 \leq P_{0, n} \leq P_{\max }$ and $0 \leq \alpha_{n} \leq 1$, which make a direct application of DDPG challenging. In the following, problem $\mathrm{P1}$ will be decomposed into two simpler optimization subproblems, in order to facilitate the application of DDPG. First, similar to [8], we introduce an energy fluctuation parameter, which is defined as the difference between the energy consumed and the energy harvested at $t_{n}$ :

$$
\bar{E}_{n}=\left(1-\alpha_{n}\right) T \eta P_{n}\left|h_{n, 0}\right|^{2}-\alpha_{n} T P_{0, n} .
$$

$\bar{E}_{n}$ can be interpreted as the energy surplus (or deficit) at $t_{n}$ if $\bar{E}_{n}>0$ (or $\bar{E}_{n}<0$ ). We note that for a fixed $\bar{E}_{n}$, the data rate at $t_{n}, R_{n}$, depends on the power allocation coefficient and the time-sharing parameter at $t_{n}$ only, where the parameters for the other time slots, i.e., $\alpha_{m}$ and $P_{0, m}, m \neq n$, will not have any impact on $R_{n}$. This observation can be clearly illustrated by recasting problem $\mathrm{P} 1$ in the following equivalent form [30, Page 133]:

$$
\begin{aligned}
& \max _{\pi_{\bar{E}_{n}}} \mathcal{E}\left\{\sum_{n=1}^{\infty} \gamma^{n-1} R_{n} \mid \pi_{\bar{E}_{n}}, s_{1}\right\} \\
& \text { s.t. } E_{n+1}=\min \left\{E_{\max }, E_{n}+\bar{E}_{n}\right\},
\end{aligned}
$$

where the subscript of policy $\pi_{\bar{E}_{n}}$ highlights the fact that the secondary user's action at $t_{n}$ is to choose $\bar{E}_{n}$ (instead of $P_{0, n}$ ), $\alpha_{n}$, and $\tilde{R}_{n}$ is defined as follows:

$$
\tilde{R}_{n}=\sup \left\{\left.R_{n}\left|\left(1-\alpha_{n}\right) T \eta P_{n}\right| h_{n, 0}\right|^{2}-\alpha_{n} T P_{0, n}=\bar{E}_{n},(\underline{\mathrm{P} 1 \mathrm{c}}),(\underline{\mathrm{P} 1 \mathrm{~d}}),(\mathrm{P} 1 \mathrm{e})\right\} .
$$


Therefore, problem $\mathrm{P} 1$ can be solved by first solving the following optimization problem:5

$$
\begin{aligned}
\max _{P_{0, n}, \alpha_{n}} & R_{n} \\
\text { s.t. } & \left(1-\alpha_{n}\right) T \eta P_{n}\left|h_{n, 0}\right|^{2}-\alpha_{n} T P_{0, n}=\bar{E}_{n} \\
& (\underline{\mathrm{P} 1 \mathrm{c}},(\underline{\mathrm{P} 1 \mathrm{~d}}),(\mathbf{\mathrm { P } 1 \mathrm { e }} .
\end{aligned}
$$

Denote the optimal solutions of problem $\mathrm{P} 3$ by $\alpha_{n}^{*}\left(\bar{E}_{n}\right)$ and $P_{0, n}^{*}\left(\bar{E}_{n}\right)$, where $\alpha_{n}^{*}\left(\bar{E}_{n}\right)$ and $P_{0, n}^{*}\left(\bar{E}_{n}\right)$ are expressed as functions of $\bar{E}_{n}$ in order to highlight the fact that the optimal solutions, $\alpha_{n}$ and $P_{0, n}$, are obtained for a given $\bar{E}_{n}$. The closed-form expressions for $\alpha_{n}^{*}\left(\bar{E}_{n}\right)$ and $P_{0, n}^{*}\left(\bar{E}_{n}\right)$ are then substituted in problem $\mathrm{P} 2$, which yields the following optimization problem: 6

$$
\begin{aligned}
& \max _{\pi_{\bar{E}_{n}}} \mathcal{E}\left\{\sum_{n=1}^{\infty} \gamma^{n-1} R_{n}\left(\alpha_{n}^{*}\left(\bar{E}_{n}\right), P_{0, n}^{*}\left(\bar{E}_{n}\right)\right) \mid \pi_{\bar{E}_{n}}, s_{1}\right\} \\
& \text { s.t. } \quad E_{n+1}=\min \left\{E_{\max }, E_{n}+\bar{E}_{n}\right\} .
\end{aligned}
$$

where $R_{n}\left(\alpha_{n}^{*}\left(\bar{E}_{n}\right), P_{0, n}^{*}\left(\bar{E}_{n}\right)\right)$ is a function of $\bar{E}_{n}$ only, i.e., $R_{n}\left(\alpha_{n}^{*}\left(\bar{E}_{n}\right), P_{0, n}^{*}\left(\bar{E}_{n}\right)\right)=$ $\alpha^{*}\left(\bar{E}_{n}\right) \ln \left(1+\frac{P_{0, n}^{*}\left(\bar{E}_{n}\right)\left|g_{0, n}\right|^{2}}{1+P_{n}\left|h_{n}\right|^{2}}\right)$.

In Section IV, problem P3 will be solved via convex optimization, where closed-form expressions for $\alpha_{n}^{*}\left(\bar{E}_{n}\right)$ and $P_{0, n}^{*}\left(\bar{E}_{n}\right)$ will be presented. In Section $\mathrm{V}$, problem $\mathrm{P} 4$ will be reformulated as a reinforcement learning problem and the DDPG algorithm will be applied to find the solution.

${ }^{5}$ For the subproblem shown in $\mathrm{P3}$ only the variables for $t_{n}$ are involved, i.e., the secondary user's data rate at $t_{n}$ is maximized by optimizing its transmit power $P_{0, n}$ and its time-sharing parameter $\alpha_{n}$ at $t_{n}$. Therefore, only the causal CSI assumption is require, and the optimal solution of problem $\mathrm{P3}$ is applicable regardless of whether the channels are time-varying or contant.

${ }^{6} \mathrm{We}$ note that P4 will be solved by applying DRL which is well known for its applicability in time-varying environments. As a result, the proposed DRL scheme can be directly applied if the channels are time-varying, as demonstrated in Section VI Furthermore, we note that the use of DRL to solve P4 requires causal CSI only, since the principle of DRL is to generate a decision by sensing the current state of the environment and using the past experience, where the knowledge of the environments in the future is not needed. 


\section{Finding Closed-Form Expressions for $\alpha_{n}^{*}\left(\bar{E}_{n}\right)$ ANd $P_{0, n}^{*}\left(\bar{E}_{n}\right)$}

In order to find closed-form expressions for $\alpha_{n}^{*}\left(\bar{E}_{n}\right)$ and $P_{0, n}^{*}\left(\bar{E}_{n}\right)$, we first recast ( $(\underline{\mathrm{P} 3})$ as follows:

$$
\begin{aligned}
\max _{P_{0, n}, \alpha_{n}} & f_{0}\left(P_{0, n}, \alpha_{n}\right) \triangleq \alpha_{n} \ln \left(1+\frac{P_{0, n}\left|g_{0, n}\right|^{2}}{1+P_{n}\left|h_{n}\right|^{2}}\right) \\
\text { s.t. } & f_{1}\left(P_{0, n}, \alpha_{n}\right)=0, \\
& f_{2}\left(P_{0, n}, \alpha_{n}\right) \leq 0,
\end{aligned}
$$

(P1d), (P1e).

where $f_{1}\left(P_{0, n}, \alpha_{n}\right)=\left(1-\alpha_{n}\right) T \eta P_{n}\left|h_{n, 0}\right|^{2}-\alpha_{n} T P_{0, n}-\bar{E}_{n}$ and $f_{2}\left(P_{0, n}, \alpha_{n}\right)=\alpha_{n} T P_{0, n}-E_{n}$.

Remark 4: Note that problem $\mathrm{P} 5$ is not jointly convex in $P_{0, n}$ and $\alpha_{n}$. For example, its equality constraint, $(\underline{\mathrm{P} 5 \mathrm{~b}})$, is not an affine function. A similar optimization problem was considered in [8], where the equality constraint was relaxed to an inequality constraint. However, in the expression for the relaxed inequality constraint shown in [8, Eq. (13)], there is still a term which involves the multiplication of two optimization variables, which means that the inequality function is not convex and hence the relaxed problem is not in a convex form.

According [30, Page 133], an equivalent form of problem ( 1 5 ) can be found as follows:

$$
\begin{array}{ll}
\max _{\alpha_{n}} & \tilde{f}_{0}\left(\alpha_{n}\right) \\
\text { s.t. } & 0 \leq \alpha_{n} \leq 1,
\end{array}
$$

where $\tilde{f}_{0}\left(\alpha_{n}\right)$ is defined as follows:

$$
\tilde{f}_{0}\left(\alpha_{n}\right)=\sup \left\{f_{0}\left(P_{0, n}, \alpha_{n}\right) \mid f_{1}\left(P_{0, n}, \alpha_{n}\right)=0, f_{2}\left(P_{0, n}, \alpha_{n}\right) \leq 0, P_{0, n} \geq 0\right\} .
$$

Finding $\tilde{f}_{0}\left(\alpha_{n}\right)$ is equivalent to solving the following optimization problem:

$$
\begin{array}{ll}
\max _{P_{0, n}} & \alpha_{n} \ln \left(1+\frac{P_{0, n}\left|g_{0, n}\right|^{2}}{1+P_{n}\left|h_{n}\right|^{2}}\right) \\
\text { s.t. } & P_{0, n}=\frac{\left(1-\alpha_{n}\right) \eta P_{n}\left|h_{n, 0}\right|^{2}}{\alpha_{n}}-\frac{\bar{E}_{n}}{\alpha_{n} T} \\
& P_{0, n} \leq \frac{E_{n}}{\alpha_{n} T} \\
& 0 \leq P_{0, n} \leq P_{\max } .
\end{array}
$$


Note that problem P7 is a function of $P_{0, n}$ only, where $\alpha_{n}$ is fixed. By using the equality constraint in ( $\mathrm{P7b})$, the optimal value of $\tilde{f}_{0}\left(\alpha_{n}\right)$ can be found as follows:

$$
\tilde{f}_{0}^{*}\left(\alpha_{n}\right)=\alpha_{n} \ln \left(1+\frac{\frac{\left(1-\alpha_{n}\right) \eta P_{n}\left|h_{n, 0}\right|^{2}\left|g_{0, n}\right|^{2}}{\alpha_{n}}-\frac{\bar{E}_{n}\left|g_{0, n}\right|^{2}}{\alpha_{n} T}}{1+P_{n}\left|h_{n}\right|^{2}}\right),
$$

where the constraints in $(\overline{\mathrm{P7C}})$ and $(\overline{\mathrm{P7d}})$ can be guaranteed by expressing the domain of $\tilde{f}_{0}\left(\alpha_{n}\right)$ as follows:

$$
\mathcal{D}=\left\{\alpha_{n} \mid 0 \leq \frac{\left(1-\alpha_{n}\right) \eta P_{n}\left|h_{n, 0}\right|^{2}}{\alpha_{n}}-\frac{\bar{E}_{n}}{\alpha_{n} T} \leq \min \left\{P_{\max }, \frac{E_{n}}{\alpha_{n} T}\right\}\right\} .
$$

By using $\tilde{f}_{0}^{*}\left(\alpha_{n}\right)$ and showing the constraints on the domain of the function explicitly, problem (P6) can be equivalently recast as follows:

$$
\begin{array}{ll}
\max _{\alpha_{n}} & \alpha_{n} \ln \left(1+\frac{\frac{\left(1-\alpha_{n}\right) \eta P_{n}\left|h_{n, 0}\right|^{2}\left|g_{0, n}\right|^{2}}{\alpha_{n}}-\frac{\bar{E}_{n}\left|g_{0, n}\right|^{2}}{\alpha_{n} T}}{1+P_{n}\left|h_{n}\right|^{2}}\right) \\
\text { s.t. } & \alpha_{n} \leq 1-\frac{\bar{E}_{n}}{T \eta P_{n}\left|h_{n, 0}\right|^{2}} \\
& \alpha_{n} \geq 1-\frac{E_{n}+\bar{E}_{n}}{T \eta P_{n}\left|h_{n, 0}\right|^{2}} \\
& \alpha_{n} \geq \frac{T \eta P_{n}\left|h_{n, 0}\right|^{2}-\bar{E}_{n}}{T \eta P_{n}\left|h_{n, 0}\right|^{2}+T P_{\max }} \\
& 0 \leq \alpha_{n} \leq 1 .
\end{array}
$$

Note that problem $\mathrm{P} 8$ involves three lower bounds and two upper bounds on $\alpha_{n}$. In addition, the domain of the objective function in (P8a) also imposes a constraint on the choice of $\alpha_{n}$. Therefore, the problem might be infeasible if one of the lower bounds is larger than one of the upper bounds, and hence it is important to carry out a feasibility study for problem P8, as is done in the following proposition.

Proposition 1. Problem P8 is always feasible.

Proof. See Appendix @.

Remark 5: For the case $\bar{E}_{n}=T \eta P_{n}\left|h_{n, 0}\right|^{2}$, the upper bound in (P8b) becomes 0, which means that the only feasible solution for this case is $\alpha_{n}^{*}\left(\bar{E}_{n}\right)=0$. Therefore, no data transmission happens and $R_{n}=0$ for this case. We note that the case with $\bar{E}_{n}=T \eta P_{n}\left|h_{n, 0}\right|^{2}$ can cause a singularity issue when analyzing the convexity of the objective function. Therefore, unless otherwise stated, it is assumed that $\bar{E}_{n} \neq T \eta P_{n}\left|h_{n, 0}\right|^{2}$ for the remainder of this section. 
Another important step in finding the optimal solution of problem $\mathrm{P} 8$ is to study the convexity of its objective function, which is done in the following proposition.

Proposition 2. The objective function of problem $P 8$ is a concave function of $\alpha_{n}$, for $\alpha_{n} \geq 0$.

Proof. See Appendix B.

Given the two useful properties provided in Propositions 1 and 2 , it can be easily verified that problem $\mathrm{P8}$ is a concave problem; however, finding a closed-form solution for problem $\mathrm{P8}$ is challenging, due to the multiple constraints on the choices of $\alpha_{n}$ as shown in $(\overline{\mathrm{P} 8 \mathrm{~b}}),(\overline{\mathrm{P} 8 \mathrm{c}}),(\overline{\mathrm{P} 8 \mathrm{~d}})$, and ( $(\mathrm{P} 8 \mathrm{e})$. It is important to point out that these constraints cannot be merged. For example, the constraint in ( $\left(\underline{\mathrm{P} 8 \mathrm{C})}\right.$ is not always stricter than $\alpha_{n} \geq 0$ shown in (P8) since

$$
1-\frac{E_{n}+\bar{E}_{n}}{T \eta P_{n}\left|h_{n, 0}\right|^{2}}=\frac{T \eta P_{n}\left|h_{n, 0}\right|^{2}-E_{n}-\bar{E}_{n}}{T \eta P_{n}\left|h_{n, 0}\right|^{2}}
$$

which can be either negative or positive. As a result, finding the optimal solution by directly applying the Karush-Kuhn-Tucker (KKT) conditions to problem $\mathrm{P8}$ is difficult, since five dual variables are required due to the large number of inequality constraints. Furthermore, the fact that the root of the first-order derivative of the objective function is in the form of the Lambert W function with two branches makes it more challenging to find a closed-form expression for the optimal solution.

Nevertheless, by using the properties of the inequality constraints of problem $\mathrm{P}$, a closed-form optimal solution can be found, as shown in the following lemma.

Lemma 1. The optimal solution for problem P8 can be expressed as follows:

$$
\alpha_{n}^{*}\left(\bar{E}_{n}\right)=\min \left\{1, \max \left\{x^{*}, \theta_{0}\right\}\right\}
$$

if $\bar{E}_{n} \neq \operatorname{T\eta P} P_{n}\left|h_{n, 0}\right|^{2}$, otherwise $\alpha_{n}^{*}\left(\bar{E}_{n}\right)=0$, where $x^{*}=\frac{\kappa_{1}-\kappa_{2}}{e^{W_{0}\left(e^{-1}\left(\kappa_{1}-1\right)\right)+1}-1+\kappa_{1}}, \theta_{0}=$ $\max \left\{1-\frac{E_{n}+\bar{E}_{n}}{T \eta P_{n}\left|h_{n, 0}\right|^{2}}, \frac{T \eta P_{n}\left|h_{n, 0}\right|^{2}-\bar{E}_{n}}{T \eta P_{n}\left|h_{n, 0}\right|^{2}+T P_{\max }}\right\}, \quad \kappa_{1}=\frac{\eta P_{n}\left|h_{n, 0}\right|^{2}\left|g_{0, n}\right|^{2}}{1+P_{n}\left|h_{n}\right|^{2}}, \quad \kappa_{2}=\frac{\bar{E}_{n}\left|g_{0, n}\right|^{2}}{T\left(1+P_{n}\left|h_{n}\right|^{2}\right)}$, and $W_{0}(\cdot)$ denotes the principle branch of the Lambert $W$ function [31].

Proof. See Appendix C.

Remark 6: Given the closed-form expression for $\alpha_{n}^{*}\left(\bar{E}_{n}\right)$, the optimal power allocation coefficient is given by

$$
P_{0, n}^{*}\left(\bar{E}_{n}\right)=\frac{\left(1-\alpha_{n}^{*}\right) \eta P_{n}\left|h_{n, 0}\right|^{2}}{\alpha_{n}^{*}}-\frac{\bar{E}_{n}}{\alpha_{n}^{*} T},
$$


if $\bar{E}_{n} \neq T \eta P_{n}\left|h_{n, 0}\right|^{2}$. We note that the case of $\bar{E}_{n}=T \eta P_{n}\left|h_{n, 0}\right|^{2}$ is not discussed in the proof of Lemma 1, As discussed in Remark 5, if $\bar{E}_{n}=T \eta P_{n}\left|h_{n, 0}\right|^{2}$, the entire time available will be used for energy harvesting, and there is no data transmission, i.e., $\alpha_{n}^{*}\left(\bar{E}_{n}\right)=0$. For this special case, there is no need to specify the value of $P_{0, n}^{*}\left(\bar{E}_{n}\right)$.

\section{A DDPG ApPROACH TO Optimize $\bar{E}_{n}$}

\section{A. A Brief Introduction to DDPG}

The ultimate goal of DDPG is to determine an action, denoted by $a$, which can maximize the action-value function, denoted by $Q(s, a)$, for a given state, denoted by $s$, via the following maximization problem [23]:

$$
a^{*}(s)=\arg \max _{a} \quad Q(s, a),
$$

which is similar to tabular reinforcement learning algorithms, such as Q-learning and stateaction-reward-state-action (SARSA) [32]. But unlike Q-learning and SARSA, for DDPG, there is no need to build a table to store the values of $Q(s, a)$. Instead, the action-value function is approximated by using neural networks, which is similar to deep Q networks (DQN). Furthermore, unlike Q-learning, SARSA, and DQN, DDPG is designed for the case when actions are continuous variables.

In particular, the four neural networks used for DDPG are listed in the following [28]:

- An actor network (also termed a policy network), parameterized by $\omega_{\mu}$, takes $s$ as its input and outputs an action which is denoted by $\mu\left(s \mid \omega_{\mu}\right)$.

- A target actor network, parameterized by $\omega_{\mu_{t}}$, outputs $\mu_{t}\left(s \mid \omega_{\mu_{t}}\right)$.

- A critic network (also termed a Q network), parameterized by $\omega_{c}$, takes $s$ and $a$ as its inputs, and outputs the corresponding state-value function, denoted by $Q\left(s, a \mid \omega_{c}\right)$.

- A target critic network, parameterized by $\omega_{c_{t}}$, outputs $Q_{t}\left(s, a \mid \omega_{c_{t}}\right)$.

The key features of DDPG are described in the following.

1) Exploration: From the user's perspective, the actor network is the most important component since it provides the desired solution. In order to encourage the algorithm to explore the environment, noise is added to the output of the actor network, which means that the action to be taken for state $s$ is given by [28]

$$
a(s)=\mu\left(s \mid \omega_{\mu}\right)+n,
$$

where $n$ denotes the exploration noise. 
2) Updating the networks: While only the actor network is used to generate the needed action, the other three networks are crucial to make sure that the actor network is properly trained. Assume that there exists a tuple $\left(s, a, r, s_{-}\right)$, where $r$ is the reward if action $a$ is taken for the current state $s$ and $s_{-}$denotes the next state. Based on this tuple, the networks are updated as follows [28]:

- The actor network is trained by maximizing the state-value function, as shown in (14). By using the parameters of the actor and critic networks, the objective function for the maximization problem can be rewritten as $J\left(\omega_{\mu}\right)=Q\left(s, a=\mu\left(s \mid \omega_{\mu}\right) \mid \omega_{c}\right)$. Given the fact that the action space is continuous and also assuming that the state-value function is differentiable, the parameters of the actor network, $\omega_{\mu}$, can be updated by carrying out gradient ascent. Note that gradient search requires the derivative of the objective function with respect to $\omega_{\mu}$, where the following chain rule can be used:

$$
\nabla \omega_{\mu} J\left(\omega_{\mu}\right)=\nabla_{a} Q\left(s, a \mid \omega_{c}\right) \nabla_{\omega_{\mu}} \mu\left(s \mid \omega_{\mu}\right) .
$$

Therefore, the output of the actor network can be used as the input of the critic network, and the parameters of the actor network $\left(\omega_{\mu}\right)$ are updated by maximizing the output of the critic network and fixing the parameters of the critic network.

- The critic network plays a crucial role in updating the actor network, as can be seen from (16). The update of the critic network relies on the two target networks. On the one hand, by using the output of the target actor network as an input of the target critic network, a target value for the state-value function is obtained as follows:

$$
y=r+\gamma Q_{t}\left(s_{-}, \mu_{t}\left(s_{-} \mid \omega_{\mu_{t}}\right) \mid \omega_{c_{t}}\right)
$$

where $\gamma$ denotes the discount parameter. On the other hand, another estimate for the statevalue function can be generated by using the critic network, i.e., $Q\left(s, a \mid \omega_{c}\right)$. The critic network can be updated by minimizing the loss defined as follows:

$$
L=\left(y-Q\left(s, a \mid \omega_{c}\right)\right)^{2} .
$$

- The two target networks have the same structure as their counterparts and their parameters are updated as follows:

$$
\omega_{c_{t}} \rightarrow \tau \omega_{c}+(1-\tau) \omega_{c_{t}}, \quad \omega_{\mu_{t}} \rightarrow \tau \omega_{\mu}+(1-\tau) \omega_{\mu_{t}}
$$

where $\tau$ is the soft updating parameter. We note that the two target networks are updated with a much lower frequency than their counterparts. 
3) Replay Buffer: Similar to DQN, for DDPG, the past experience, i.e., multiple tuples from the past, $\left(s_{i}, a_{i}, r_{i}, s_{i,-}\right)$, are stored in a pool, termed the replay buffer. When the networks are updated, a fixed number of the tuples are randomly selected from the buffer and used for network updating, which means that both (16) and (18) are carried out in a batch mode.

\section{B. Application of DDPG to CR-NOMA}

By using the closed-form expression for $\alpha^{*}\left(\bar{E}_{n}\right)$ developed in Lemma 1 and $P_{0, n}^{*}\left(\bar{E}_{n}\right)$ shown in (13), the long-term throughput maximization problem can be rewritten as follows:

$$
\begin{aligned}
& \max _{\pi_{\bar{E}_{n}}} \mathcal{E}\left\{\sum_{n=1}^{\infty} \gamma^{n-1} \alpha^{*}\left(\bar{E}_{n}\right) \ln \left(1+\frac{P_{0, n}^{*}\left(\bar{E}_{n}\right)\left|g_{0, n}\right|^{2}}{1+P_{n}\left|h_{n}\right|^{2}}\right) \mid \pi_{\bar{E}_{n}}, s_{1}\right\} \\
& \text { s.t. } \quad E_{n+1}=\min \left\{E_{\max }, E_{n}+\bar{E}_{n}\right\} .
\end{aligned}
$$

Because problem $\mathrm{P9}$ is a function of a single continuous variable, $\bar{E}_{n}$, it is ideally suited for the application of DDPG. The key step for the application of reinforcement learning is to define the state space, the action space, and the reward, as discussed in the following.

1) State space: The state space consists of all possible states. As discussed in Section ஹ, for the considered long-term system throughput maximization problem, a natural choice for the state is $s_{n}=\left[\begin{array}{lll}\left|g_{0, n}\right|^{2} & \left|h_{n}\right|^{2} \quad\left|h_{n, 0}\right|^{2} \quad E_{n}\end{array}\right]^{T}$, which includes the channel gains associated with the primary user served at $t_{n}$, the secondary user's channel to the base station, and the energy available at the beginning of $t_{n}$.

2) Action Space: The action space consists of all possible actions taken by the secondary user. For the considered throughput maximization problem, it is natural to use $\bar{E}_{n}$ as the action. For example, if both $\left|h_{n}\right|^{2}$ and $\left|h_{n, 0}\right|^{2}$ are extremely strong, a good choice for the action is $\bar{E}_{n} \geq 0$, such that data transmission is avoided due to the strong interference caused by $\overline{\mathrm{U}}_{n}$, and to spend more time for energy harvesting due to the strong connection between $\bar{U}_{n}$ and $\mathrm{U}_{0}$.

However, it is important to point out that the range of $\bar{E}_{n}$ can be quite large. In particular, as shown in the proof of Proposition 1, the value of $\bar{E}_{n}$ is bounded by the two following extreme situations:

$$
\underbrace{-\min \left\{E_{n}, T P_{\max }\right\}}_{\text {No energy harvesting }} \leq \bar{E}_{n} \leq \underbrace{\min \left\{E_{\max }-E_{n}, T \eta P_{n}\left|h_{n, 0}\right|^{2}\right\}}_{\text {No data transmission }},
$$

which can be a very large negative number and a very large positive number, respectively. Note that, compared to the existing work in [8], where only $-E_{n}$ is used as the lower bound on 
$\bar{E}_{n}$, we also include $-T P_{\max }$ in the lower bound. This is due to the transmit power constraint $P_{0, n} \leq P_{\max }$, which means that the maximal energy consumed within $T$ seconds is $T P_{\max }$, if there is sufficient energy available at the beginning of $t_{n}$, i.e., $E_{n}>T P_{\max }$.

In order to improve the stability of the used neural networks, it is preferable to constrain the possible choices of the action within a small and fixed range, ideally between 0 and 1 . By using the upper and lower bounds on $\bar{E}_{n}$ and introducing an auxiliary variable $\beta_{n}, 0 \leq \beta_{n} \leq 1, \bar{E}_{n}$ can be expressed as follows:

$$
\bar{E}_{n}=\beta_{n} \min \left\{E_{\max }-E_{n}, T \eta P_{n}\left|h_{n, 0}\right|^{2}\right\}-\left(1-\beta_{n}\right) \min \left\{E_{n}, T P_{\max }\right\}
$$

It is straightforward to verify that the lower bound in (20) is used if $\beta_{n}=0$, and the upper bound can be realized by letting $\beta_{n}=1$. Therefore, $\beta$ is a suitable action variable for DDPG networks.

3) Reward: Because the objective function of the optimization problem in P9 is based on the secondary user's long-term data rate, it is natural to use $R_{n}$ as the reward at $t_{n}$.

By using the defined state space, action space, and reward, DDPG can be applied straightforwardly. In particular, each episode consists of multiple steps/iterations. During each step, the DDPG algorithm first generates an action according to the current state, finds the next state according to the chosen action, and then the DDPG agent starts learning by updating the four neural networks as discussed in the previous subsection. The details for the DDPG implementation of DDPG can be found in [33].

\section{Simulation Results}

In this section, we study the performance of the proposed DDPG assisted NOMA transmission strategy by using computer simulation results. In our simulations, the learning rates for the actor and critic networks are set as 0.002 and 0.004, respectively. The reward discount parameter is $\gamma=0.9$, the network updating parameter is $\tau=0.01$, and the batch size for the replay experience is 32 . The base station is located at the origin of the $x-y$ plane, and the secondary user is located at $(1 \mathrm{~m}, 1 \mathrm{~m})$. The primary users' transmit power is set as $P_{n}=30 \mathrm{dBm}$, the initial energy in the battery is $E_{\max }=0.1 \mathrm{~J}, T=1 \mathrm{~s}, P_{\max }=0.1 \mathrm{~W}$, and the energy harvesting efficiency coefficient is set as $\eta=0.7$. The additive white Gaussian noise power spectral density is -170 $\mathrm{dBm} / \mathrm{Hz}$, the total bandwidth is $1 \mathrm{MHz}$, the carrier frequency is $914 \mathrm{MHz}$, the path loss model in [34] is used, and the path loss exponent is 3 . 
Regarding the neural networks, a simple neural network which consists of 2 hidden layers with $N_{n}=64$ nodes each is used for the actor network, where the rectified linear activation function (ReLU) is used in the first hidden layer, and the hyperbolic tangent function is used for the second hidden layer and the output layer. Since the critic network takes two inputs, $s$ and $a$, both $s$ and $a$ are fed to two individual hidden layers with 64 nodes each before they are concatenated and connected to another hidden layer with 64 nodes. ReLU is used in all the layers of the critic network. The two target networks are built in the same manner as their counterparts. The detailed setups for the four employed neural networks can be found in [33]. We note that the use of DDPG results in a higher computational complexity compared to the two benchmark schemes outlined in Remark 2. Take the greedy scheme as an example, which uses all available energy for data transmission, and hence is computationally efficient to implement. For DDPG, if the used simple actor network is fully trained, generating $\bar{E}_{n}$ given $s_{n}$ requires a moderate computational complexity of $\mathcal{O}\left(N_{n}^{2}\right)$, since the numbers of inputs, outputs, and layers are much smaller than $N_{n}$, where $\mathcal{O}(\cdot)$ denotes the computational complexity operator [35]. The complexity analysis of the training stage is challenging not only because it depends on how many gradient iterations are carried out but also because the employed critic network is not fully connected. Due to space limitations, a full complexity analysis and methods to reduce complexity will be treated as an important direction for future research. It is expected that the training stage of the DDPG entails a high computational complexity, where a promising energy and computationally efficient mitigation approach is to apply mobile edge computing (MEC) and allow the secondary user to offload computations to the base station [36].

The concepts of steps, episodes, and experiments for DDPG can be interpreted in the considered CR-NOMA context as follows. Each step represents a time slot, each episode consists of 100 time slots (or steps), and each experiment consists of multiple episodes. At the beginning of each experiment, the neural networks are randomly initialized. At the beginning of each episode, the secondary user's battery is reset to $E_{\max }$.

\section{A. A Two-User Deterministic Case}

In order to gain insight into the performance of DDPG, we first focus on a deterministic case with two primary users, where the two primary users are located at $(0 \mathrm{~m}, 1 \mathrm{~m})$ and $(0 \mathrm{~m}, 1000$ $\mathrm{m})$, only large-scale path loss is considered, and random fading is omitted. The rationale behind this setting is the study of an extreme scenario, in which $\mathrm{U}_{1}$ has a strong channel to both the 


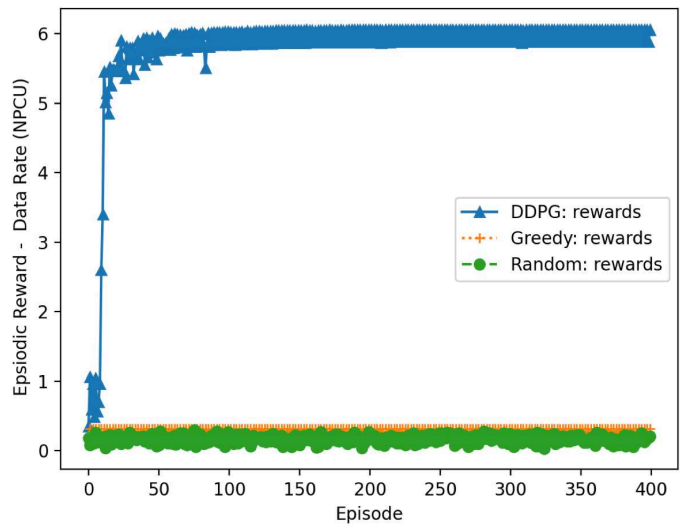

(a) Experiment I

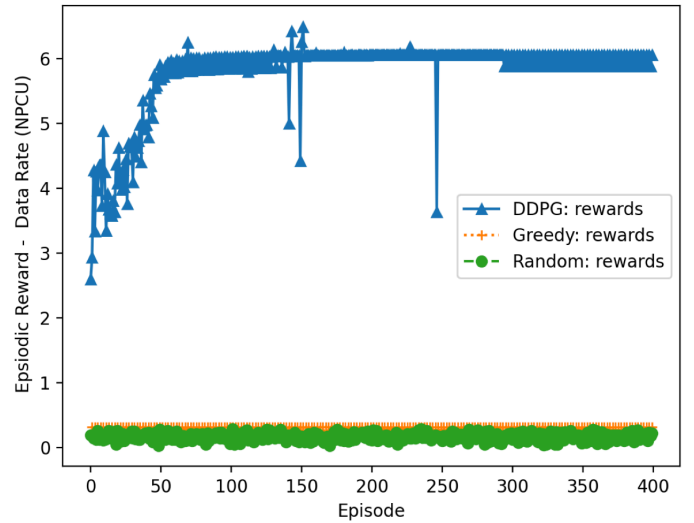

(b) Experiment II

Fig. 2. A deterministic extreme case with $M=2$ primary users. The two primary users are located at $(0 \mathrm{~m}, 1 \mathrm{~m})$ and $(0 \mathrm{~m}$, $1000 \mathrm{~m}$ ). Only large-scale path loss is considered and random fading is omitted. The difference between the two experiments is caused by the fact that the neural networks are randomly initialized at the beginning of each experiment.

base station and $\mathrm{U}_{0}$, but $\mathrm{U}_{2}$ 's channels to both nodes are extremely weak. An intuitive decision for this case is to ask $\mathrm{U}_{0}$ to carry out energy harvesting only, when $\mathrm{U}_{1}$ transmits, which has the following two benefits: severe interference from $U_{1}$ is avoided and a large amount of energy from $\mathrm{U}_{1}$ is harvested. When $\mathrm{U}_{2}$ transmits, an intuitive decision is to avoid energy harvesting at $\mathrm{U}_{0}$ since only a small amount of energy can be harvested due to the severe path loss between $\mathrm{U}_{0}$ and $\mathrm{U}_{2}$. Instead, $\mathrm{U}_{0}$ should carry out data transmission only, since $\mathrm{U}_{2}$ will not cause much interference. With this intuitive decision, the amount of energy harvested when $\mathrm{U}_{1}$ transmits is given by [34]

$$
E=\left(1-\alpha_{n}\right) T \eta P_{n}\left|h_{n, 0}\right|^{2}=0.7 / 10^{3.17} \mathrm{~J}=7 * 10^{-4.17} \mathrm{~J} \approx 4.7 * 10^{-4} \mathrm{~J} .
$$

When $\mathrm{U}_{2}$ transmits, the entire time slot is used for data transmission using the harvested energy shown in (22). Therefore, the available transmit power is $\frac{E}{T}=4.7 * 10^{-4} \mathrm{~W}$, which means that the following average data rate, denoted by $\bar{R}_{n}$, is achievable by the secondary user:

$$
\begin{aligned}
\bar{R}_{n} & =\frac{1}{2} \alpha_{n} \ln \left(1+\frac{P_{0, n}\left|g_{0, n}\right|^{2}}{1+P_{n}\left|h_{n}\right|^{2}}\right) \\
& =\frac{1}{2} \ln \left(1+\frac{4.7 * 10^{-4} \times \frac{1}{10^{3.17 * 2^{\frac{3}{2}}}}}{10^{-14}+10^{-3.17} \times 1000^{-3}}\right) \approx 6.01 \mathrm{NPCU},
\end{aligned}
$$

where the factor $\frac{1}{2}$ is used since $\mathrm{U}_{0}$ transmits only when $\mathrm{U}_{2}$ transmits. Comparing the result in (24) with Fig. 2, one can observe that the proposed reinforcement learning scheme realizes this intuitive decision. We note that DDPG might perform slightly differently for different random initializations, as shown in Figs. 2(a) and 2(b), On the other hand, the benchmark schemes yield 


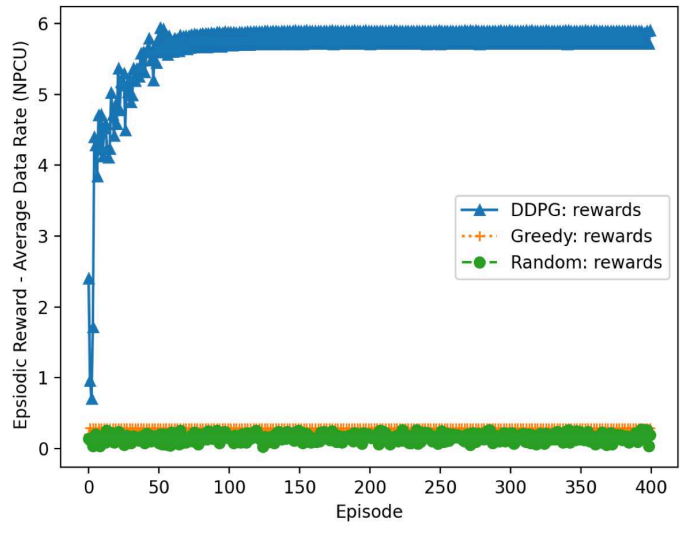

(a) Experiment I

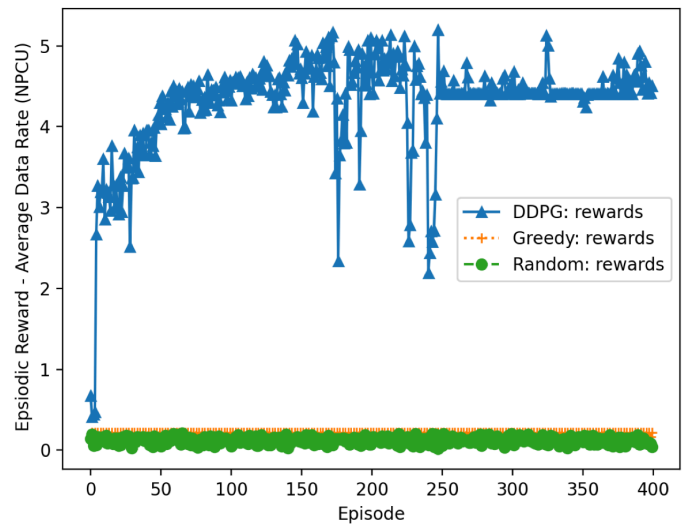

(b) Experiment II

Fig. 3. The performance of the proposed DDPG scheme with $M=2$ primary users. Both large-scale path loss and small-scale multi-path fading are considered. The two experiments are based on different small-scale fading realizations.

much worse performances than the proposed scheme, since the decisions they make are not based on a long-term objective. For example, when $U_{1}$ transmits, the greedy algorithm will still try to facilitate data transmission, which results in an inefficient energy use due to the strong interference caused by $\mathrm{U}_{1}$.

\section{B. General Multi-User Cases with Constant Fading}

In this subsection, the performance of the proposed reinforcement learning scheme is studied for a more general case with multiple users. In particular, the $M$ users are equally spaced on a segment between $(1 \mathrm{~m}, 0 \mathrm{~m})$ and $(1000 \mathrm{~m}, 0 \mathrm{~m})$. Furthermore, small-scale multi-path fading is also considered, in addition to path loss. In order to study the convergence of the proposed DDPG algorithm, during each experiment which contains multiple episodes, the users' channels

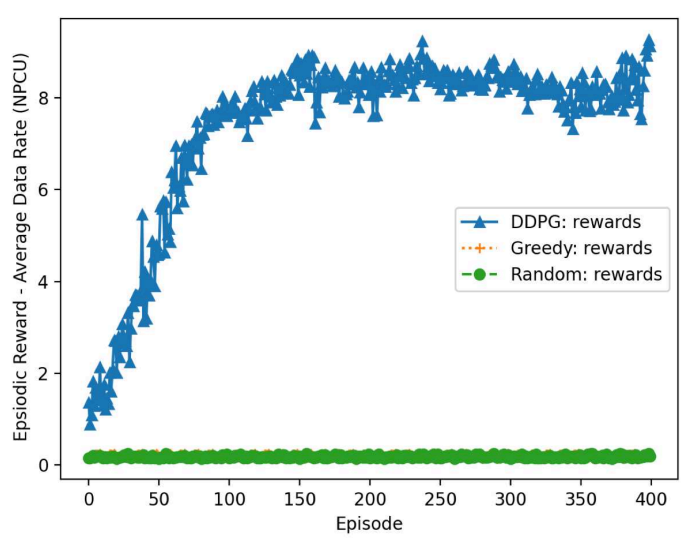

(a) Experiment I

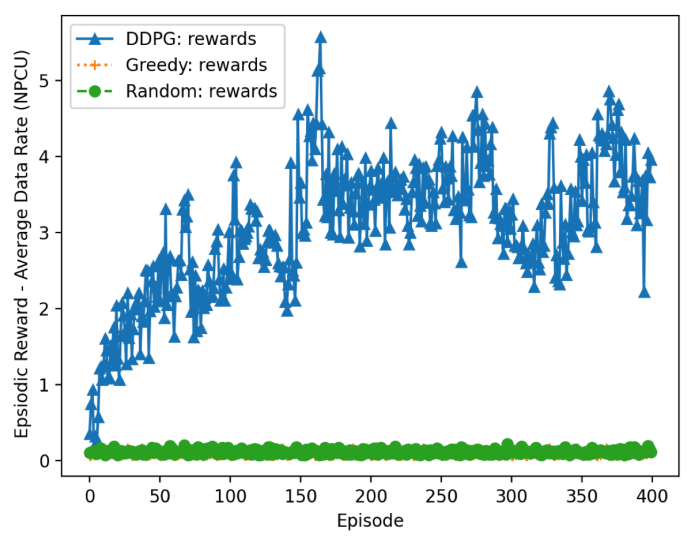

(b) Experiment II

Fig. 4. The performance of the proposed DDPG scheme with $M=10$ primary users. Both large-scale path loss and small-scale multi-path fading are considered. The two experiments are based on different small-scale fading realizations. 


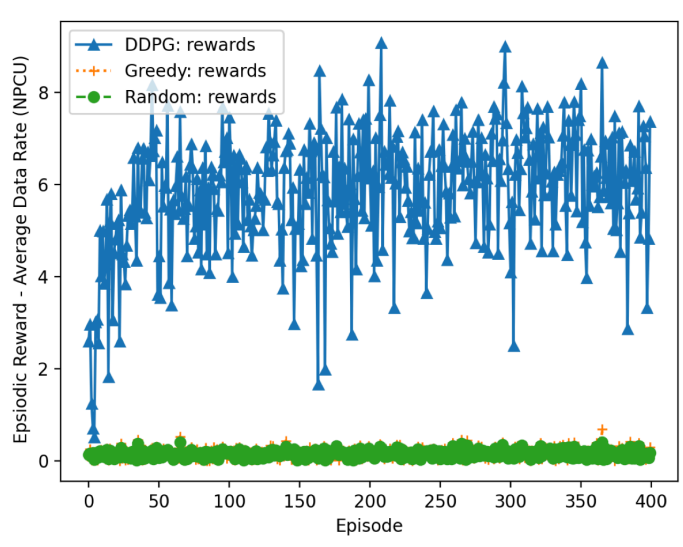

(a) $M=2$

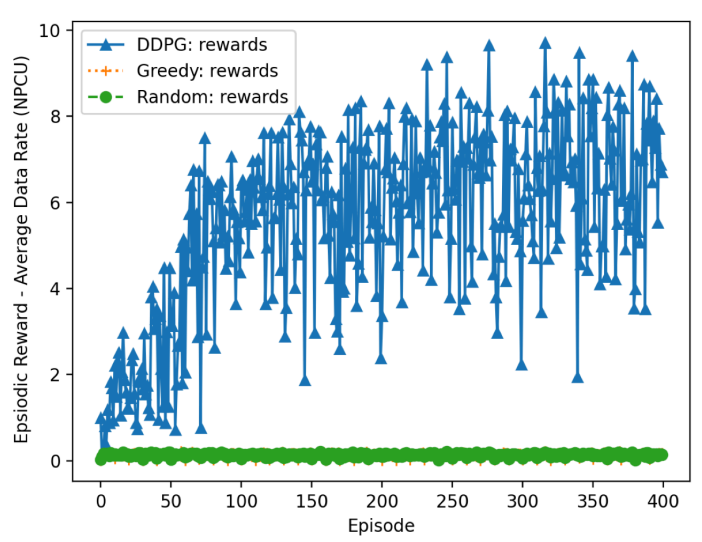

(b) $M=10$

Fig. 5. The performance of the proposed DDPG scheme in time-varying scenarios, where different small-scale fading realizations are used in different episodes.

are kept constant, where independent and identically distributed (i.i.d.) complex Gaussian random variables with zero means and unit variance are used to simulate the small-scale channel fading.

In Figs. 3 and 4, $M=2$ and $M=10$ primary users are considered, respectively. On the one hand, comparing Fig. 3 to Fig. 2, a data rate reduction can be observed, which is due to the consideration of channel fading. On the other hand, comparing 4(a) to Fig. 3, it is interesting to observe that the case with $M=10$ can yield a larger data rate than the case with $M=2$. This is due to the fact that for $M>2$, it is possible for the secondary user to use more than half of the time for data transmission. As can be observed by comparing the respective subfigures of Fig. 3 and 4, different data rates are realized for a given $M$, which is due to the fact that the subfigures employ different realizations of the small-scale fading. However, regardless of the realizations of the random fading, the proposed DDPG scheme can always achieve a considerably larger data rate than the two benchmark schemes, as is evident from the figures.

Recall that for the deterministic case with $M=2$ considered in Fig. 2, the decision generated by the proposed DDPG scheme converges quickly, e.g., after 50 episodes the DDPG curves shown in Fig. 2 become almost flat. This property disappears in general for the cases with random fading. In particular, depending on the random fading realization, the DDPG agent might lead to a constant decision policy after a sufficiently large number of episodes, as shown in Figs. 3(a) and 4(a). However, Figs. 3(b) and 4(b) also show that for certain random fading realizations, the DDPG algorithm may converge very slowly. One possible reason for this observation is that for the two-user deterministic case considered in Fig. 2, the underlying pattern is simple, e.g., 
one user has strong channels but the other user does not. This simple pattern can be quickly and efficiently learned by DDPG. When there are more users packed in the same area and/or the fading is random, it is possible that such a clear pattern does not exist, i.e., multiple users' channel conditions are similar to each other. As a result, there is no clear strategy for energy harvesting and data transmission.

\section{General Multi-User Cases with Time-Varying Fading}

Finally, the performance of the proposed DDPG scheme is studied in a general multi-user scenario with time-varying channels. For the simulation results presented in the following, the locations of the users are fixed in the same manner as in the previous subsection, and different small-scale fading realizations are used in different episodes. In Fig. 5, the performance of the proposed DDPG algorithm is shown as a function of the number of episodes in the considered time-varying scenario. As can be observed from the figure, the use of the proposed DDPG algorithm can still offer a significant performance gain over the two benchmark schemes, particularly if a sufficient number of episodes is employed. In addition, compared to the case $M=2$, the case $M=10$ requires more episodes to ensure a significant performance gain over the benchmark schemes. These observations are consistent with those made for time-invariant channels. We note that the episode reward curves in Fig. 5 show more variation than those for the time-invariant case. This is mainly due to the fact that different fading realizations are experienced in different episodes. As a consequence, the maximal throughputs in different episodes are also expected to be different, which means that the episode reward curves cannot be flat, even if the proposed algorithm can achieve the respective maximal throughputs.

In Fig. 6, the performances of the three considered schemes are compared by using the average data rate as the metric, where Monte Carlo simulations are carried out to average out the randomness caused by time-varying fading. In Fig. 6(a), three different choices for the number of episodes are used, in order to illustrate how the performance of the DDPG scheme is affected by the amount of training. As can be observed from Fig. 6(a), even for a single episode, the proposed DDPG algorithm can already realize a larger data rate than the greedy and random schemes. By increasing the number of episodes from 1 to 10 and 20, the performance gain of the proposed DDPG algorithm over the benchmark schemes can be further improved, as shown in Fig. 6(a), Recall that we observed from Figs. 3 and 4 that the proposed DDPG algorithm starts to converge after 100 episodes. This is the reason why increasing the number of episodes 


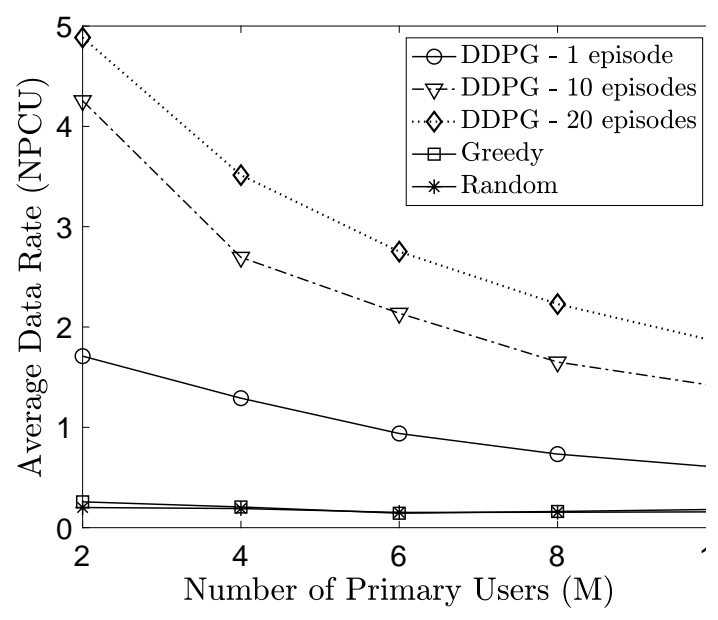

(a) The cases with 1,10 , and 20 episodes

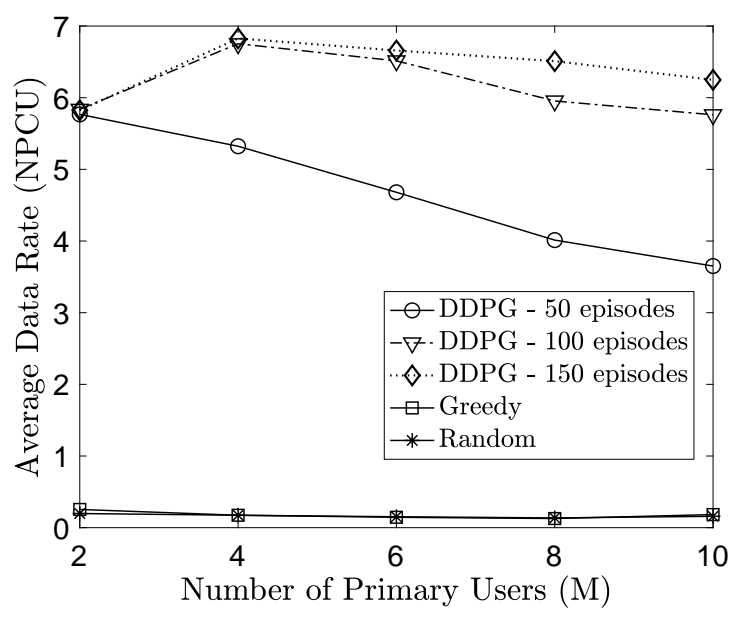

(b) The cases with 50, 100, and 150 episodes

Fig. 6. Impact of $M$ and the number of the used episodes on the performance of the proposed DDPG scheme in time-varying scenarios, where different small-scale fading realizations are used in different episodes.

in Fig. 6(b) from 100 to 150 improves the performance of the proposed DDPG scheme only slightly. Another important observation from Fig. 6 is that the case with more primary users participating in the NOMA transmission requires a larger amount of training. This observation is expected since a case with more primary users is more complex and hence more episodes are required to train the DDPG algorithm.

\section{CONCLUSIONS}

In this paper, machine learning has been applied in CR-NOMA networks to facilitate spectrum and energy cooperation between multiple primary users and an energy-constrained secondary user. The goal of the machine learning algorithm is to maximize the secondary user's long-term throughput, which is challenging due to the need for making decisions which yield long-term gains but might result in short-term losses. For example, when, in a given time slot, a primary user with large channel gains transmits, an intuitive approach is that the secondary user should not carry out data transmission but perform energy harvesting only, which results in zero data rate for this time slot but yields benefits in the long-term. Here, the considered long-term throughput maximization problem was first reformulated, and then a DRL approach, termed DDPG, was applied to emulate the intuitive solution. The provided simulation results demonstrate that the proposed deep reinforcement learning assisted NOMA transmission scheme can yield significant performance gains over two benchmark schemes.

While the case with a single secondary user has been the focus of this paper, the proposed idea of spectrum and energy sharing among primary and secondary users can be extended to scenarios with multiple secondary users. For example, the scheme in [37] shows the possibility 
of carrying out spectrum and energy cooperation among one primary downlink user and multiple secondary uplink users. Compared to the case with a single secondary user, the resource allocation design for multi-user systems is more challenging, due to the constraint that additional secondary users should be served without affecting the primary users' QoS experience. In addition, the interference between the secondary users has to be carefully controlled. Furthermore, in this paper, a linear energy harvesting model has been assumed, and the impact of the energy consumed for signal processing and by the radio frequency circuits has not been taken into consideration. These assumptions have allowed us to gain important insights regarding the longterm optimization of the two conflicting tasks, energy harvesting and data transmission. However, the extension of the proposed machine learning algorithm to the case with more practical assumptions is an important direction for future research. Moreover, it was assumed in this paper that the energy constrained secondary user carries out radio-frequency energy harvesting. Alternatively, the secondary user can also use backscatter communications (BackCom) to realize battery-less transmission, and the application of DRL to BackCom assisted NOMA transmission is another interesting direction for future research [38].

\section{APPENDIX A \\ PROOF FOR PROPOSITION 1}

In order to analyze the feasibility of problem $\mathrm{P} 8$, the range of $\bar{E}_{n}$ needs to be studied first. Recall that $\bar{E}_{n}$ is the difference between the energy harvested and consumed at $t_{n}$, i.e., $(1-$ $\left.\alpha_{n}\right) T \eta P_{n}\left|h_{n, 0}\right|^{2}-\alpha_{n} T P_{0, n}$. The smallest value achievable by $\bar{E}_{n}$ is caused by the case with no energy harvesting, i.e., all the time is used for data transmission and $\alpha_{n}=1$, which means that

$$
\bar{E}_{n} \geq-T P_{0, n}
$$

Furthermore, the energy available at the beginning of $t_{n}$ is $E_{n}$, which means that the energy available for data transmission is capped by $E_{n}$, which results in another lower bound on $\bar{E}_{n}$, i.e., $\bar{E}_{n} \geq-E_{n}$. As a result, $\bar{E}_{n}$ can be lower bounded as follows:

$$
\bar{E}_{n} \geq-\min \left\{E_{n}, T P_{\max }\right\}
$$

where we replaced $P_{0, n}$ by $P_{\max }$ in (25). On the other hand, the largest value achievable by $\bar{E}_{n}$ is caused by the decision not to carry out any data transmission but perform energy harvesting only, i.e., $\alpha_{n}=0$. In this case, an upper bound on $\bar{E}_{n}$ can be obtained as follows:

$$
\bar{E}_{n} \leq T \eta P_{n}\left|h_{n, 0}\right|^{2}
$$


Moreover, there is an upper bound on the amount of energy stored at $t_{n}$ because of the finite battery capacity. In particular, given the capacity of the battery, $E_{\max }$, and the energy available in the battery at the beginning of $t_{n}, E_{n}$, the maximal amount of new energy that can be stored at $t_{n}$ is capped by $E_{\max }-E_{n}$. As a result, $\bar{E}_{n}$ is upper bounded as follows:

$$
\bar{E}_{n} \leq \min \left\{E_{\max }-E_{n}, T \eta P_{n}\left|h_{n, 0}\right|^{2}\right\} .
$$

By combining (26) and (28), $\bar{E}_{n}$ can be bounded as follows:

$$
-\min \left\{E_{n}, T P_{\max }\right\} \leq \bar{E}_{n} \leq \min \left\{E_{\max }-E_{n}, T \eta P_{n}\left|h_{n, 0}\right|^{2}\right\}
$$

The feasibility of problem $\mathrm{P} 8$ is illustrated in the following two steps. The first step is to show that the lower bounds on $\alpha_{n}$ for problem $\mathrm{P} 8$ do not conflict with the upper bounds. The second step is to show that the intersection of the set defined by the constraints of problem $\mathrm{P} 8$ and the domain of its objective function is non-empty.

Note that there are three lower bounds defined by $(\underline{\mathrm{P} 8 \mathrm{c}}),(\underline{\mathrm{P} 8 \mathrm{~d}})$, and $(\overline{\mathrm{P} 8 \mathrm{e}})$, respectively. Firstly, we focus on the lower bound $\alpha_{n} \geq 0$ in ( $\mathrm{P8}$. Apparently, it does not conflict with the upper bound in ( $(\mathrm{P} 8 \mathrm{e})$, i.e., $\alpha_{n} \leq 1$. In order to compare this lower bound to the upper bound in ( $(\mathrm{P} 8 \mathrm{~b})$, $\alpha_{n} \leq 1-\frac{\bar{E}_{n}}{T \eta P_{n}\left|h_{n, 0}\right|^{2}}$, we first rewrite the upper bound in (29) as follows:

$$
\bar{E}_{n} \leq \min \left\{E_{\max }-E_{n}, T \eta P_{n}\left|h_{n, 0}\right|^{2}\right\} \leq T \eta P_{n}\left|h_{n, 0}\right|^{2} .
$$

Therefore, $1-\frac{\bar{E}_{n}}{T \eta P_{n}\left|h_{n, 0}\right|^{2}} \geq 0$, and hence one can conclude that the lower bound $\alpha_{n} \geq 0$ does not conflict with the upper bound in ( $\mathrm{P8b}$.

Secondly, we focus on the lower bound in ( $\overline{\mathrm{P} 8 \mathrm{c}}), \alpha_{n} \geq 1-\frac{E_{n}+\bar{E}_{n}}{T \eta P_{n}\left|h_{n, 0}\right|^{2}}$. To prove that it does not conflict with the upper bound in (P8), $\alpha_{n} \leq 1$, it is sufficient to show the following

$$
1-\frac{E_{n}+\bar{E}_{n}}{T \eta P_{n}\left|h_{n, 0}\right|^{2}} \leq 1,
$$

which requires $\bar{E}_{n} \geq-E_{n}$. This always holds because the use of the lower bound in (29) yields $\bar{E}_{n} \geq-\min \left\{E_{n}, T P_{\max }\right\}$ or equivalently $\bar{E}_{n} \geq \max \left\{-E_{n},-T P_{\max }\right\} \geq-E_{n}$. In addition, it is straightforward to show that the lower bound in (P8C) is always smaller than the upper bound in (P8b) by using the fact that $E_{n} \geq 0$.

Thirdly, we focus on the lower bound in ( $(\overline{\mathrm{P} 8 \mathrm{~d}}), \alpha_{n} \geq \frac{T \eta P_{n}\left|h_{n, 0}\right|^{2}-\bar{E}_{n}}{T \eta P_{n}\left|h_{n, 0}\right|^{2}+T P_{\max }}$. To prove that it does not conflict with the upper bound in (P8), it is sufficient to show the following

$$
\frac{T \eta P_{n}\left|h_{n, 0}\right|^{2}-\bar{E}_{n}}{T \eta P_{n}\left|h_{n, 0}\right|^{2}+T P_{\max }} \leq 1,
$$


which requires $\bar{E}_{n} \geq-T P_{\max }$. This also always holds because the use of the bound in (29) yields $\bar{E}_{n} \geq \max \left\{-E_{n},-T P_{\max }\right\} \geq-T P_{\max }$. In order to show that the lower bound in (P8d) is always smaller than the upper bound in $(\overline{\mathrm{P} 8 \mathrm{~b}})$, we need to prove the following inequality

$$
\frac{T \eta P_{n}\left|h_{n, 0}\right|^{2}-\bar{E}_{n}}{T \eta P_{n}\left|h_{n, 0}\right|^{2}+T P_{\max }} \leq 1-\frac{\bar{E}_{n}}{T \eta P_{n}\left|h_{n, 0}\right|^{2}}
$$

which can be simplified as follows:

$$
\bar{E}_{n} \leq T \eta P_{n}\left|h_{n, 0}\right|^{2}
$$

This inequality always holds since $\bar{E}_{n} \leq \min \left\{E_{\max }-E_{n}, T \eta P_{n}\left|h_{n, 0}\right|^{2}\right\} \leq T \eta P_{n}\left|h_{n, 0}\right|^{2}$ based on (29). In summary, the constraints of problem (P8) do not conflict with each other, and the set defined by these constraints is not empty.

The last step to analyse the feasibility of problem $\mathrm{P8}$ is to show that the intersection of the domain of the objective function of problem $\mathrm{P8}$ and the set defined by the constraints is not empty. Recall that the domain of the objective function (P8a) imposes the following constraint on $\alpha_{n}$ :

$$
1+\frac{\left(1-\alpha_{n}\right)}{\alpha_{n}} \frac{\eta P_{n}\left|h_{n, 0}\right|^{2}\left|g_{0, n}\right|^{2}}{1+P_{n}\left|h_{n}\right|^{2}}-\frac{1}{\alpha_{n}} \frac{\bar{E}_{n}\left|g_{0, n}\right|^{2}}{T\left(1+P_{n}\left|h_{n}\right|^{2}\right)} \geq 0 .
$$

As can be shown, the constraint imposed on $\alpha_{n}$ by problem $\mathrm{P} 8$ is stricter than (35). In particular, one can convert the following inequality

$$
\frac{\left(1-\alpha_{n}\right)}{\alpha_{n}} \frac{\eta P_{n}\left|h_{n, 0}\right|^{2}\left|g_{0, n}\right|^{2}}{1+P_{n}\left|h_{n}\right|^{2}}-\frac{1}{\alpha_{n}} \frac{\bar{E}_{n}\left|g_{0, n}\right|^{2}}{T\left(1+P_{n}\left|h_{n}\right|^{2}\right)} \geq 0,
$$

into the following equivalent form:

$$
\left(1-\alpha_{n}\right) T \eta P_{n}\left|h_{n, 0}\right|^{2}-\bar{E}_{n} \geq 0 .
$$

With some algebraic manipulations, one can find that the inequality constraint in (37) is equivalent to constraint $(\overline{\mathrm{P} 8 \mathrm{~b}})$. In other words, the intersection of the set defined by the constraints of problem $\mathrm{P8}$ and the domain of its objective function is non-empty. As a result, problem ( is always feasible, and the proposition is proved.

\section{APPENDIX B}

\section{PROOF FOR PROPOSITION 2}

Recall that the objective function of problem $\mathrm{P8}$ can be expressed as follows:

$$
\tilde{f}_{0}^{*}\left(\alpha_{n}\right)=\alpha_{n} \ln \left(1+\frac{\left(1-\alpha_{n}\right)}{\alpha_{n}} \frac{\eta P_{n}\left|h_{n, 0}\right|^{2}\left|g_{0, n}\right|^{2}}{1+P_{n}\left|h_{n}\right|^{2}}-\frac{1}{\alpha_{n}} \frac{\bar{E}_{n}\left|g_{0, n}\right|^{2}}{T\left(1+P_{n}\left|h_{n}\right|^{2}\right)}\right) .
$$


By using (37) and the fact that $T \eta P_{n}\left|h_{n, 0}\right|^{2} \geq \bar{E}_{n}$, it can be shown that $\alpha_{n}=0$ is in the domain of $\tilde{f}_{0}^{*}\left(\alpha_{n}\right)$.

To simplify notations, we define $\kappa_{1}=\frac{\eta P_{n}\left|h_{n, 0}\right|^{2}\left|g_{0, n}\right|^{2}}{1+P_{n}\left|h_{n}\right|^{2}}, \kappa_{2}=\frac{\bar{E}_{n}\left|g_{0, n}\right|^{2}}{T\left(1+P_{n}\left|h_{n}\right|^{2}\right)}$, and $x=\alpha_{n}$. Therefore, the objective function $\tilde{f}_{0}^{*}(x)$ can be expressed as follows:

$$
\begin{aligned}
\tilde{f}_{0}^{*}(x) & =x \ln \left(1+\frac{1-x}{x} \kappa_{1}-\frac{1}{x} \kappa_{2}\right) \\
& =x \ln \left(1-\kappa_{1}+\frac{\kappa_{1}-\kappa_{2}}{x}\right) .
\end{aligned}
$$

As discussed in the proof of Proposition 1, the term $1+\frac{1-x}{x} \kappa_{1}-\frac{1}{x} \kappa_{2}$ is strictly positive once all the constraints in problem $\mathrm{P} 8$ are satisfied. Furthermore, as discussed in Remark 5, it is assumed that $\bar{E}_{n} \neq T \eta P_{n}\left|h_{n, 0}\right|^{2}$. Therefore, the first order derivative of $\tilde{f}_{0}^{*}(x)$ is given by

$$
\begin{aligned}
\frac{d \tilde{f}_{0}^{*}(x)}{d x} & =\ln \left(1-\kappa_{1}+\frac{\kappa_{1}-\kappa_{2}}{x}\right)-\frac{\frac{\kappa_{1}-\kappa_{2}}{x}}{1-\kappa_{1}+\frac{\kappa_{1}-\kappa_{2}}{x}} \\
& =\ln \left(1-\kappa_{1}+\frac{\kappa_{1}-\kappa_{2}}{x}\right)-\frac{\left(\kappa_{1}-\kappa_{2}\right)}{x\left(1-\kappa_{1}\right)+\kappa_{1}-\kappa_{2}} .
\end{aligned}
$$

The second order derivative of $\tilde{f}_{0}^{*}(x)$ is given by

$$
\frac{d^{2} \tilde{f}_{0}^{*}(x)}{d x^{2}}=-\frac{\left(\kappa_{1}-\kappa_{2}\right)}{x\left(x\left(1-\kappa_{1}\right)+\kappa_{1}-\kappa_{2}\right)}+\frac{\left(1-\kappa_{1}\right)\left(\kappa_{1}-\kappa_{2}\right)}{\left(x\left(1-\kappa_{1}\right)+\kappa_{1}-\kappa_{2}\right)^{2}} .
$$

With some algebraic manipulations, the second order derivative can be expressed as follows:

$$
\frac{d^{2} \tilde{f}_{0}^{*}(x)}{d x^{2}}=\frac{-\left(\kappa_{1}-\kappa_{2}\right)^{2}}{\left(x\left(1-\kappa_{1}\right)+\kappa_{1}-\kappa_{2}\right)^{2} x} \leq 0,
$$

for $x \geq 0$. Therefore, the objective function is a concave function for $\alpha_{n} \geq 0$ and the proof is complete.

\section{Appendix C}

\section{PROOF FOR LEMMA 1}

Problem $\mathrm{P8}$ can be expressed in the following compact form

$$
\begin{array}{ll}
\max _{\alpha_{n}} & \alpha_{n} \ln \left(1+\frac{\left(1-\alpha_{n}\right)}{\alpha_{n}} \kappa_{1}-\frac{1}{\alpha_{n}} \kappa_{2}\right) \\
\text { s.t. } & \alpha_{n} \leq \min \left\{1,1-\frac{\bar{E}_{n}}{T \eta P_{n}\left|h_{n, 0}\right|^{2}}\right\} \\
& \alpha_{n} \geq \max \left\{0,1-\frac{E_{n}+\bar{E}_{n}}{T \eta P_{n}\left|h_{n, 0}\right|^{2}}, \frac{T \eta P_{n}\left|h_{n, 0}\right|^{2}-\bar{E}_{n}}{T \eta P_{n}\left|h_{n, 0}\right|^{2}+T P_{\max }}\right\} .
\end{array}
$$


Denote $x^{*}$ as the optimal solution of the maximization problem $\max _{x \geq 0} \tilde{f}_{0}^{*}(x)$. As indicated by Proposition $2, \tilde{f}_{0}^{*}(x)$ is a concave function of $x$ for $x \geq 0$, and hence there exists a single maximum, i.e., $x^{*}$. However, because $x^{*}$ is obtained by discarding the constraints, (P10b and $(\mathrm{P} 10 \mathrm{c})$, it is possible that $x^{*}$ is not a feasible solution of problem $\mathrm{P} 10$.

Therefore, the lemma is proved in the following three steps. In the first step, we analyze the properties of $\tilde{f}_{0}^{*}(x)$ at the boundary values given by $(\underline{\mathrm{P} 10 \mathrm{~b}})$ and $(\underline{\mathrm{P} 10 \mathrm{c})}$. In the second step, a closed-form expression for $x^{*}$ is developed, where the conclusion about the comparison between $x^{*}$ and one of the boundary values, $1-\frac{\bar{E}_{n}}{T \eta P_{n}\left|h_{n, 0}\right|^{2}}$, is crucial to avoid the ambiguity caused by the two branches of the Lambert $\mathrm{W}$ function. In the third step, a closed-form solution for problem P10 is obtained by using the obtained closed-form $x^{*}$.

1) Analysis of the properties of $\tilde{f}_{0}^{*}(x)$ : The aim of this subsection is to show that $\tilde{f}_{0}^{*}(x)$ is non-decreasing at $x=0$ and non-increasing at $1-\frac{\bar{E}_{n}}{T \eta P_{n}\left|h_{n, 0}\right|^{2}}$, the boundary values shown in $(\underline{\mathrm{P} 10 \mathrm{~b}})$ and $(\mathrm{P} 10 \mathrm{c})$, which can be proved by showing the following

$$
f^{\prime}(0) \geq 0 \quad \& \quad f^{\prime}\left(1-\frac{\bar{E}_{n}}{T \eta P_{n}\left|h_{n, 0}\right|^{2}}\right) \leq 0,
$$

where $f^{\prime}(x) \triangleq \frac{d \tilde{f}_{0}^{*}(x)}{d x}$ denotes the first order derivative of $\tilde{f}_{0}^{*}(x)$. We note that (43) requires the implicit assumption that both 0 and $1-\frac{\bar{E}_{n}}{T \eta P_{n}\left|h_{n, 0}\right|^{2}}$ are in the domain of the objective function, which can be easily shown by using (37).

In order to show $f^{\prime}(0) \geq 0$, assume $x=\epsilon \rightarrow 0$. Thus, by using (40), $f^{\prime}(\epsilon)$ can be expressed as follows:

$$
\begin{aligned}
f^{\prime}(\epsilon) & =\ln \left(1-\kappa_{1}+\frac{\kappa_{1}-\kappa_{2}}{\epsilon}\right)-\frac{\left(\kappa_{1}-\kappa_{2}\right)}{\epsilon\left(1-\kappa_{1}\right)+\kappa_{1}-\kappa_{2}} \\
& \rightarrow \ln \left(1-\kappa_{1}+\frac{\kappa_{1}-\kappa_{2}}{\epsilon}\right)-1 .
\end{aligned}
$$

Note that $\kappa_{1}-\kappa_{2} \geq 0$, which can be proved as follows:

$$
\begin{aligned}
\kappa_{1}-\kappa_{2} & =\frac{\eta P_{n}\left|h_{n, 0}\right|^{2}\left|g_{0, n}\right|^{2}}{1+P_{n}\left|h_{n}\right|^{2}}-\frac{\bar{E}_{n}\left|g_{0, n}\right|^{2}}{T\left(1+P_{n}\left|h_{n}\right|^{2}\right)} \\
& =\frac{\left(T \eta P_{n}\left|h_{n, 0}\right|^{2}-\bar{E}_{n}\right)\left|g_{0, n}\right|^{2}}{T\left(1+P_{n}\left|h_{n}\right|^{2}\right)} \geq 0,
\end{aligned}
$$

which is due to the fact that $\bar{E}_{n} \leq T \eta P_{n}\left|h_{n, 0}\right|^{2}$ as shown in (29). As a result, $\frac{\kappa_{1}-\kappa_{2}}{\epsilon}$ approaches positive infinity for $\epsilon \rightarrow 0$, where the trivial case $\bar{E}_{n}=T \eta P_{n}\left|h_{n, 0}\right|^{2}$ is not considered as discussed in Remark 5. Therefore, for $\epsilon \rightarrow 0, f^{\prime}(\epsilon)$ can be approximated as follows:

$$
f^{\prime}(\epsilon) \underset{\epsilon \rightarrow 0}{\rightarrow} \ln \left(1-\kappa_{1}+\frac{\kappa_{1}-\kappa_{2}}{\epsilon}\right)-1 \geq 0 .
$$


In order to show $f^{\prime}\left(1-\frac{\bar{E}_{n}}{T \eta P_{n}\left|h_{n, 0}\right|^{2}}\right) \leq 0$, define $\theta_{1}=1-\frac{\bar{E}_{n}}{T \eta P_{n}\left|h_{n, 0}\right|^{2}}$ for simplicity of notation. The first order derivative $f^{\prime}\left(\theta_{1}\right)$ is given by:

$$
f^{\prime}\left(\theta_{1}\right)=\ln \left(1-\kappa_{1}+\frac{\kappa_{1}-\kappa_{2}}{\theta_{1}}\right)-\frac{\left(\kappa_{1}-\kappa_{2}\right)}{\theta_{1}\left(1-\kappa_{1}\right)+\kappa_{1}-\kappa_{2}} .
$$

The following identity holds:

$$
\frac{\kappa_{1}-\kappa_{2}}{\theta_{1}}=\frac{\left(T \eta P_{n}\left|h_{n, 0}\right|^{2}-\bar{E}_{n}\right)\left|g_{0, n}\right|^{2}}{T\left(1+P_{n}\left|h_{n}\right|^{2}\right)} \frac{T \eta P_{n}\left|h_{n, 0}\right|^{2}}{T \eta P_{n}\left|h_{n, 0}\right|^{2}-\bar{E}_{n}}=\frac{\eta P_{n}\left|h_{n, 0}\right|^{2}\left|g_{0, n}\right|^{2}}{\left(1+P_{n}\left|h_{n}\right|^{2}\right)}=\kappa_{1},
$$

which means

$$
f^{\prime}\left(\theta_{1}\right)=-\frac{\left(\kappa_{1}-\kappa_{2}\right)}{\theta_{1}\left(1-\kappa_{1}\right)+\kappa_{1}-\kappa_{2}} .
$$

$f^{\prime}\left(\theta_{1}\right)$ can be further rewritten as follows:

$$
f^{\prime}\left(\theta_{1}\right)=-\frac{\frac{\left(\kappa_{1}-\kappa_{2}\right)}{\theta_{1}}}{\left(1-\kappa_{1}\right)+\frac{\kappa_{1}-\kappa_{2}}{\theta_{1}}}=-\frac{\kappa_{1}}{\left(1-\kappa_{1}\right)+\kappa_{1}}=-\kappa_{1} \leq 0,
$$

since $\kappa_{1}$ is non-negative. Therefore, the inequality in (43) is proved.

By using the concavity of the objective function and also (43), it is straightforward to show that $x^{*}$ is bounded as follows:

$$
0 \leq x^{*} \leq \theta_{1}
$$

The bounds in (50) will be useful to solve an ambiguity issue caused by the Lambert $\mathrm{W}$ function, as shown in the following subsection.

2) Finding a closed-form expression for $x^{*}$ : The fact that the objective function $\tilde{f}_{0}^{*}(x)$ is concave means that $x^{*}$ is the root of the following equation:

$$
\ln \left(1-\kappa_{1}+\frac{\kappa_{1}-\kappa_{2}}{x^{*}}\right)-\frac{\left(\kappa_{1}-\kappa_{2}\right)}{x^{*}\left(1-\kappa_{1}\right)+\kappa_{1}-\kappa_{2}}=0,
$$

which can be rewritten as follows:

$$
\ln \left(1-\kappa_{1}+\frac{\kappa_{1}-\kappa_{2}}{x^{*}}\right)-\frac{\frac{\left(\kappa_{1}-\kappa_{2}\right)}{x^{*}}}{\left(1-\kappa_{1}\right)+\frac{\kappa_{1}-\kappa_{2}}{x^{*}}}=0 .
$$

Define $y^{*}=1-\kappa_{1}+\frac{\kappa_{1}-\kappa_{2}}{x^{*}}$, which leads to:

$$
\ln \left(y^{*}\right)-\frac{\left(y^{*}-1+\kappa_{1}\right)}{y^{*}}=0
$$

or equivalently

$$
y^{*} \ln \left(y^{*}\right)-y^{*}=\kappa_{1}-1
$$


In order to apply the the Lambert $\mathrm{W}$ function, define $y^{*}=e^{z^{*}}$, which results in the following equation:

$$
z^{*} e^{z^{*}}-e^{z^{*}}=\kappa_{1}-1
$$

or equivalently

$$
\left(z^{*}-1\right) e^{z^{*}-1}=e^{-1}\left(\kappa_{1}-1\right)
$$

The form of (56) makes the application of the Lambert $\mathrm{W}$ function possible. Recall that there is a single real-valued solution for the equation $w e^{w}=z$, if $z \geq 0$, which is given by $w=W_{0}(z)$, i.e., the principal branch of the Lambert $\mathrm{W}$ function. However, if $z$ is negative, there are two real-valued solutions, denoted by $w=W_{0}(z)$ and $w=W_{-1}(z)$, respectively.

For the considered problem, $\kappa_{1}-1$ is not always positive since

$$
\kappa_{1}-1=\frac{\eta P_{n}\left|h_{n, 0}\right|^{2}\left|g_{0, n}\right|^{2}}{1+P_{n}\left|h_{n}\right|^{2}}-1
$$

can be negative if $\left|h_{n}\right|^{2}$ is much larger than $\left|h_{n, 0}\right|^{2}$. As a result, for the case $\kappa_{1}-1<0$, there are two real-valued solutions corresponding to the two branches of the Lambert W function as follows:

$$
\begin{aligned}
& z^{*}-1=W_{0}\left(e^{-1}\left(\kappa_{1}-1\right)\right), \\
& z^{*}-1=W_{-1}\left(e^{-1}\left(\kappa_{1}-1\right)\right) .
\end{aligned}
$$

With the two possible choices for $z^{*}$, there will be also two choices for $x^{*}$, which makes the final expression of $\alpha^{*}(\bar{E})_{n}$ very complicated. Fortunately, the solution corresponding to $z^{*}-1=W_{-1}\left(e^{-1}\left(\kappa_{1}-1\right)\right)$ can be discarded, as proved in the following.

In the following, assume $\kappa_{1}-1<0$, and proof by contradiction will be used to show that $z^{*}-1=W_{-1}\left(e^{-1}\left(\kappa_{1}-1\right)\right)$ is not a feasible solution. Note that, according to (57), $\frac{\eta P_{n}\left|h_{n, 0}\right|^{2}\left|g_{0, n}\right|^{2}}{1+P_{n}\left|h_{n}\right|^{2}}$ is strictly positive, and hence $\kappa_{1}-1$ is strictly larger than -1 , i.e. $-1<\kappa_{1}-1<0$, According to the property of the Lambert $\mathrm{W}$ function, $w=W_{-1}(z)<-1$, for $-1<z<0$. Therefore, $z^{*}-1=W_{-1}\left(e^{-1}\left(\kappa_{1}-1\right)\right)<-1$,which means

$$
z^{*}<0 \Longrightarrow y^{*}=e^{z^{*}}<1 \text {. }
$$

By using the relationship between $y^{*}$ and $x^{*}, x^{*}$ needs to satisfy the following inequality:

$$
1-\kappa_{1}+\frac{\kappa_{1}-\kappa_{2}}{x^{*}}<1 \Longrightarrow \kappa_{1}\left(1-x^{*}\right)<\kappa_{2},
$$


where the fact that $x^{*} \geq 0$ is used. By substituting the expressions of $\kappa_{1}$ and $\kappa_{2}$ into the above inequality, $x^{*}$ needs to satisfy the following inequality:

$$
\frac{\eta P_{n}\left|h_{n, 0}\right|^{2}\left|g_{0, n}\right|^{2}}{1+P_{n}\left|h_{n}\right|^{2}}\left(1-x^{*}\right)<\frac{\bar{E}_{n}\left|g_{0, n}\right|^{2}}{T\left(1+P_{n}\left|h_{n}\right|^{2}\right)},
$$

which can be further rewritten as follows:

$$
T \eta P_{n}\left|h_{n, 0}\right|^{2}\left(1-x^{*}\right)<\bar{E}_{n}
$$

In the following, we show that the condition in (63) cannot be met. According to our previous analysis, $f^{\prime}\left(\theta_{1}\right) \leq 0$, which implies the following:

$$
x^{*} \leq \theta_{1} \triangleq 1-\frac{\bar{E}_{n}}{T \eta P_{n}\left|h_{n, 0}\right|^{2}} .
$$

With some algebraic manipulations, the inequality that $x^{*}$ needs to satisfy can be expressed as follows:

$$
\left(1-x^{*}\right) T \eta P_{n}\left|h_{n, 0}\right|^{2} \geq \bar{E}_{n}
$$

which contradicts the condition in (63). Therefore, the solution $z^{*}-1=W_{-1}\left(e^{-1}\left(\kappa_{1}-1\right)\right)$ can be discarded, and $z^{*}-1=W_{0}\left(e^{-1}\left(\kappa_{1}-1\right)\right)$ is the solution which should be used.

Therefore, with $z^{*}-1=W_{0}\left(e^{-1}\left(\kappa_{1}-1\right)\right), y^{*}$ can be written as follows:

$$
y^{*}=e^{z^{*}}=e^{W_{0}\left(e^{-1}\left(\kappa_{1}-1\right)\right)+1},
$$

which means that a closed-form expression for $x^{*}$ is obtained as follows:

$$
x^{*}=\frac{\kappa_{1}-\kappa_{2}}{e^{W_{0}\left(e^{-1}\left(\kappa_{1}-1\right)\right)+1}-1+\kappa_{1}} .
$$

3) Finding the optimal solution for problem P10. Although $x^{*}$ yields the maximal value for the objective function, $x^{*}$ is not necessarily the optimal solution of problem $\mathbf{P 1 0}$, since $x^{*}$ might violate one of the constraints of problem $\mathrm{P10}$, To facilitate the discussions, the constraints of problem $\mathrm{P10}$ are rewritten as follows:

$$
\max \left\{0, \theta_{0}\right\} \leq \alpha_{n} \leq \min \left\{1, \theta_{1}\right\}
$$

which defines the feasible set of $\alpha_{n}$, where $\theta_{0}$ is defined in Lemma 1, While the relationship between $0, \theta_{1}$ and $x^{*}$ is fixed as shown in (50), the relationship between $x^{*}, 1$, and $\theta_{0}$ is not fixed, and there are three possible cases which yield three different solutions:

- Case 1: When $\max \left\{0, \theta_{0}\right\} \leq x^{*}$ and $x^{*} \leq \min \left\{1, \theta_{1}\right\}, x^{*}$ is inside of the feasible set of problem $\mathrm{P} 10$ as shown in Fig. 7. Hence, $x^{*}$ is the optimal solution of the problem. 


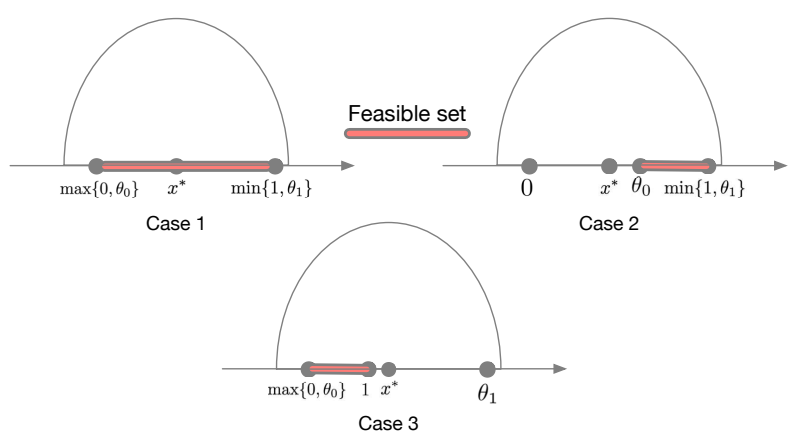

Fig. 7. Illustration for three possible cases for $x^{*}$

- Case 2: When $x^{*}<\theta_{0}$, the feasible set of $\alpha_{n}$ is $\left[\theta_{0}, \min \left\{1, \theta_{1}\right\}\right]$ and $x^{*}$ is at the left-hand side of the feasible set as shown in Fig. 7. The function is monotonically decreasing over the feasible set, and hence $\theta_{0}$ is the optimal solution of the problem.

- Case 3: When $x^{*}>1$, the feasible set of $\alpha_{n}$ becomes $\left[\max \left\{0, \theta_{0}\right\}, 1\right]$ and $x^{*}$ is at the right-hand side of the feasible set as shown in the figure. The function is monotonically increasing over the feasible set, and hence 1 is the optimal solution of the problem.

In summary, the optimal solution for problem $\mathrm{P} 10$ is given by

$$
\alpha_{n}^{*}\left(\bar{E}_{n}\right)=\min \left\{1, \max \left\{x^{*}, \theta_{0}\right\}\right\}
$$

and the lemma is proved.

\section{REFERENCES}

[1] C. Jiang, H. Zhang, Y. Ren, Z. Han, K. Chen, and L. Hanzo, "Machine learning paradigms for next-generation wireless networks," IEEE Wireless Commun., vol. 24, no. 2, pp. 98-105, Apr. 2017.

[2] C. M. Bishop, Pattern Recognition and Machine Learning. Springer, New York, USA, 2006.

[3] R. C. Daniels, C. M. Caramanis, and R. W. Heath, "Adaptation in convolutionally coded MIMO-OFDM wireless systems through supervised learning and SNR ordering," IEEE Trans. Veh. Tech., vol. 59, no. 1, pp. 114-126, Jan. 2010.

[4] H. Ye, G. Y. Li, and B. Juang, "Power of deep learning for channel estimation and signal detection in OFDM systems," IEEE Wireless Commun. Lett., vol. 7, no. 1, pp. 114-117, Feb. 2018.

[5] S. Jung, B. Moon, and D. Han, "Unsupervised learning for crowdsourced indoor localization in wireless networks," IEEE Trans. Mobile Computing, vol. 15, no. 11, pp. 2892-2906, Nov. 2016.

[6] H. Huang, W. Xia, J. Xiong, J. Yang, G. Zheng, and X. Zhu, "Unsupervised learning-based fast beamforming design for downlink MIMO,” IEEE Access, vol. 7, pp. 7599-7605, 2019.

[7] N. C. Luong, D. T. Hoang, S. Gong, D. Niyato, P. Wang, Y. Liang, and D. I. Kim, "Applications of deep reinforcement learning in communications and networking: A survey," IEEE Commun. Surveys Tuts., vol. 21, no. 4, pp. 3133-3174, Fourth quarter 2019.

[8] L. Li, H. Xu, J. Ma, A. Zhou, and J. Liu, "Joint EH time and transmit power optimization based on DDPG for EH communications," IEEE Commun. Letters, vol. 24, no. 9, pp. 2043-2046, Sept. 2020.

[9] P. Blasco, D. Gunduz, and M. Dohler, "A learning theoretic approach to energy harvesting communication system optimization," IEEE Trans. Wireless Commun., vol. 12, no. 4, pp. 1872-1882, Apr. 2013.

[10] M. Vaezi, Z. Ding, and H. V. Poor, Multiple Access Techniques for 5G Wireless Networks and Beyond. Springer International Publishing, 2019.

[11] J. Cui, Z. Ding, P. Fan, and N. Al-Dhahir, "Unsupervised machine learning-based user clustering in millimeter-wave-NOMA systems," IEEE Trans. Wireless Commun., vol. 17, no. 11, pp. 7425-7440, Nov. 2018.

[12] H. Zhang, H. Zhang, W. Liu, K. Long, J. Dong, and V. C. M. Leung, "Energy efficient user clustering, hybrid precoding and power optimization in Terahertz MIMO-NOMA systems," IEEE J. Sel. Areas Commun., vol. 38, no. 9, pp. 2074-2085, Sept. 2020. 
[13] J. Luo, J. Tang, D. K. C. So, G. Chen, K. Cumanan, and J. A. Chambers, "A deep learning-based approach to power minimization in multi-carrier NOMA with SWIPT," IEEE Access, vol. 7, pp. 17450-17 460, 2019.

[14] C. Li, H. Wang, and R. Song, "Intelligent offloading for NOMA-assisted MEC via dual connectivity," IEEE Internet of Things Journal, pp. 1-12, 2020.

[15] G. Gui, H. Huang, Y. Song, and H. Sari, "Deep learning for an effective nonorthogonal multiple access scheme," IEEE Trans. Veh. Tech., vol. 67, no. 9, pp. 8440-8450, Sept. 2018.

[16] M. Kim, N. Kim, W. Lee, and D. Cho, "Deep learning-aided SCMA,” IEEE Commun. Lett., vol. 22, no. 4, pp. 720-723, Apr. 2018.

[17] O. Maraqa, A. S. Rajasekaran, S. Al-Ahmadi, H. Yanikomeroglu, and S. M. Sait, "A survey of rate-optimal power domain NOMA with enabling technologies of future wireless networks," IEEE Commun. Surveys Tuts., vol. 22, no. 4, pp. 21922235, Fourth quarter 2020.

[18] F. Hussain, S. A. Hassan, R. Hussain, and E. Hossain, "Machine learning for resource management in cellular and IoT networks: Potentials, current solutions, and open challenges," IEEE Commun. Surveys Tuts., vol. 22, no. 2, pp. 1251-1275, Second quarter 2020.

[19] M. Vaezi, G. A. Aruma Baduge, Y. Liu, A. Arafa, F. Fang, and Z. Ding, "Interplay between NOMA and other emerging technologies: A survey," IEEE Trans. Cognitive Commun. and Networking, vol. 5, no. 4, pp. 900-919, Dec. 2019.

[20] R. He and Z. Ding, Applications of Machine Learning in Wireless Communications. IET Press, London, UK, 2019.

[21] Z. Ding, P. Fan, and H. V. Poor, "Impact of user pairing on 5G non-orthogonal multiple access," IEEE Trans. Veh. Tech., vol. 65, no. 8, pp. 6010-6023, Aug. 2016.

[22] X. Zhou, R. Zhang, and C. K. Ho, "Wireless information and power transfer: Architecture design and rate-energy tradeoff," IEEE Trans. Wirel. Commun., vol. 61, no. 11, pp. 4754-4767, Nov. 2013.

[23] R. S. Sutton and A. G. Barto, Reinforcement Learning: An Introduction. MIT Press, Cambridge, UK, 1998.

[24] L. P. Kaelbling, M. L. Littman, and A. W. Moore, "Reinforcement learning: A survey," Journal of Artificial Intelligence Research, vol. 4, pp. 237-285, Feb. 1996.

[25] G. Gui, M. Liu, F. Tang, N. Kato, and F. Adachi, "6G: Opening new horizons for integration of comfort, security, and intelligence," IEEE Wireless Commun., vol. 27, no. 5, pp. 126-132, Oct. 2020.

[26] M. Liu, T. Song, and G. Gui, "Deep cognitive perspective: Resource allocation for NOMA-based heterogeneous IoT with imperfect SIC," IEEE Internet of Things Journal, vol. 6, no. 2, pp. 2885-2894, Apr. 2019.

[27] V. Mnih, K. Kavukcuoglu, D. Silver, A. A. Rusu, J. Veness, M. G. Bellemare, A. Graves, M. Riedmiller, A. K. Fidjeland, G. Ostrovski, S. Petersen, C. Beattie, A. Sadik, I. Antonoglou, H. King, D. Kumaran, D. Wierstra, S. Legg, and D. Hassabis, "Human-level control through deep reinforcement learning," Nature, vol. 518, no. 7540, pp. 529-533, 2015.

[28] T. P. Lillicrap, J. J. Hunt, A. Pritzel, N. Heess, T. Erez, Y. Tassa, D. Silver, and D. Wierstra, "Continuous control with deep reinforcement learning," Available on-line at arXiv:1509.02971, 2015.

[29] Z. Ding, R. Schober, and H. V. Poor, "Unveiling the importance of SIC in NOMA systems: Part I - state of the art and recent findings," IEEE Commun. Lett., vol. 24, no. 11, pp. 2373-2377, Nov. 2020.

[30] S. Boyd and L. Vandenberghe, Convex Optimization. Cambridge University Press, Cambridge, UK, 2003.

[31] I. S. Gradshteyn and I. M. Ryzhik, Table of Integrals, Series and Products, 6th ed. New York: Academic Press, 2000.

[32] J. Cui, Z. Ding, Y. Deng, A. Nallanathan, and L. Hanzo, "Adaptive UAV-trajectory optimization under quality of service constraints: A model-free solution," IEEE Access, vol. 8, pp. 112 253-112 265, 2020.

[33] Python source code, Available on-line at https://github.com/zhiguo-ding/CRNOMA_DDPG.

[34] S. Y. Seidel and T. S. Rappaport, "914 MHz path loss prediction models for indoor wireless communications in multifloored buildings," IEEE Trans. Antennas Propag., vol. 40, no. 2, pp. 207-217, Feb. 1992.

[35] I. Goodfellow, Y. Bengio, A. Courville, and F. Bach, Deep Learning, 3rd ed. MIT Press, 2017.

[36] Y. Mao, C. You, J. Zhang, K. Huang, and K. B. Letaief, "A survey on mobile edge computing: The communication perspective," IEEE Commun. Surveys Tuts., vol. 19, no. 4, pp. 2322-2358, 2017.

[37] Z. Ding and H. V. Poor, "On the application of BAC-NOMA to 6G umMTC," IEEE Commun. Lett., Available on-line at arXiv:2102.06584, 2021.

[38] F. D. Ardakani and V. W. Wong, "Joint reflection coefficient selection and subcarrier allocation for backscatter systems with NOMA," in Proc. IEEE Wireless Commun. and Networking Conf. (WCNC), May, Seoul, South Korea 2020. 